\title{
Angiosperm diversity in a Lowland Semideciduous Seasonal Forest in Pernambuco State, Brazil
}

\author{
Rafaela Alves Pereira-Silva ${ }^{1 *}$, Beatriz Rayrana de Araújo Gama ${ }^{\circledR}$, Joésili Cristina Pereira de Oliveira ${ }^{2}$, \\ Jone Clebson Ribeiro Mendes ${ }^{\circledR}$, Jorge Irapuan de Souza Barbosa ${ }^{1}$, Sarah Maria Athiê-Souza ${ }^{\circledR}$, Leidiana \\ Lima dos Santos ${ }^{1}$, Margareth Ferreira de Sales ${ }^{2}$ \& Ana Luiza Du Bocage ${ }^{3}$ \\ ${ }^{1}$ Universidade Federal Rural de Pernambuco, Departamento de Biologia, CEP: 52.171-930, Recife, PE, Brasil. \\ ${ }^{2}$ Universidade Federal Rural de Pernambuco, Programa de Pós-graduação em Biodiversidade, Departamento \\ de Biologia, CEP: 52.171-930, Recife, PE, Brasil. \\ ${ }^{3}$ Instrituto Agronomico de Pernambuco, Herbário Dárdano de Andrade Lima, CEP: 1371, 50761-000, Recife, \\ PE, Brasil. \\ *Corresponding author: rafaela.news@hotmail.com \\ PEREIRA-SILVA, R.A., GAMA, B.R.A., OLIVEIRA, J.C.P., MENDES, J.C.R., BARBOSA, J.I.S., ATHIÊ- \\ SOUZA, S.M., SANTOS, L.L., SALES, M.F., BOCAGE, A.L.D. Angiosperm diversity in a Lowland \\ Semideciduous Seasonal Forest in Pernambuco State, Brazil. Biota Neotropica 22(1): e20211223. \\ https://doi.org/10.1590/1676-0611-BN-2021-1223.
}

\begin{abstract}
Angiosperm species growing in a fragment of Lowland Semideciduous Seasonal Forest in the Tapacurá Ecological Station (TES) in Pernambuco State (Brazil) were surveyed. Botanical collections to compose a species list were undertaken and these species identified by comparisons with specimens deposited in the HST, PEUFR and IPA herbaria, in addition to online database queries. A total of 479 species were identified, belonging to 81 families and 285 genera. The families with the largest numbers of species were Fabaceae (68), Rubiaceae (25), Malvaceae (20), Myrtaceae (20), Cyperaceae (19), Poaceae (19), Bromeliaceae (18), Euphorbiaceae (17), Asteraceae (16), and Sapindaceae (14), which together accounted for $49.27 \%$ of the total number of species. Great similarity was observed between our results and surveys of other lowland forests in northeastern Brazil, with the families Fabaceae, Rubiaceae, and Myrtaceae having the greatest species richness. This study added 44 new occurrences for Pernambuco State, demonstrating the richness and abundance of TES taxa and the importance of their conservation to the regional flora.

Keywords: Atlantic Forest; Conservation; Flora; Tapacurá.
\end{abstract} Diversidade de Angiospermas de uma Floresta Estacional Semidecidual de Terras Baixas em Pernambuco,
Brasil

Resumo: Angiospermas da Estação Ecológica de Tapacurá (EET), localizada em um fragmento de Floresta Estacional Semidecidual de Terras Baixas, Floresta Atlântica, foram analisadas. Coletas botânicas foram realizadas para compor uma lista de espécies e essas espécies identificadas por comparação com espécimes depositados nos herbários HST, PEUFR e IPA, além de consultas a banco de dados online. Um total de 479 espécies foram identificadas, pertencentes a 81 famílias e 285 gêneros. As famílias mais ricas foram Fabaceae (68), Rubiaceae (25), Malvaceae (20), Myrtaceae (20), Cyperaceae (19), Poaceae (19), Bromeliaceae (18), Euphorbiaceae (17), Asteraceae (16) e Sapindaceae (14), que juntas representaram 49,27\% do número total de espécies. Grande semelhança foi observada entre nossos resultados e levantamentos de outras florestas de várzea no nordeste do Brasil, com as famílias Fabaceae, Rubiaceae e Myrtaceae apresentando a maior riqueza de espécies. Este estudo adicionou 44 novas ocorrências para o Estado de Pernambuco, demonstrando a riqueza e abundância dos táxons de TES e a importância de sua conservação para a flora regional.

Palavras-chave: Mata Atlântica; Conservação; Flora; Tapacurá. 


\section{Introduction}

Seasonal Semideciduous Forest is a vegetation type of the Atlantic Forest that extends since the south of Rio Grande do Norte state until the north of Rio de Janeiro, as well as important disjunctions also occur in interior depressions such as those of the Pantanal in Mato Grosso state (IBGE 1992). The forests there are exposed to two climatic regimes: a tropical regime with a period of intense rainfall that can reach $\leq 1600 \mathrm{~mm} /$ year, followed by severe drought (a dry period that extends for approximately six months with a total precipitation $\leq 100 \mathrm{~mm}$ ); and a more subtropical regime with a dry period with cooler temperatures (IBGE 1992, Pennington et al. 2000). The seasonal semi-deciduous forests can lose between $20 \%$ and $50 \%$ of their leaves during the dry period, while seasonal deciduous forests can lose up to $50 \%$ of their leaves at that time (IBGE 1992).

The seasonal forests in northeastern Brazil occur in transition areas between the coastal Atlantic Forest and the inland Caatinga dryland vegetation, or in semiarid zones in mountain ranges (Cunha \& Silva Júnior, 2014). Those forests formations are fragmented, discontinuously distributed, and are currently surrounded by sugar cane monoculture plantations (Saccharum officinarum L.) or urban areas, so that their biodiversity is continuously and severely threatened (Damasceno-Júnior et al. 2009, Dias 2005, Pereira \& Alves 2007).

The Tapacurá Ecological Station (TES) is a conservation area conserving an Atlantic Forest remnant of predominantly seasonal semi-deciduous lowland forest (Almeida \& Oliveira 2009). The station was established as an initiative of Professor João Vasconcelos Sobrinho as an Environmental Protection Area of the Federal Rural University of Pernambuco (UFRPE) in 1975 (CPRH 2019), at the "São Bento" Escola Superior de Agricultura (1917-1936) where the UFRPE was first established (being transferred to the city of Recife in 1938) (Almeida and Oliveira 2009).

The TES was exposed to numerous anthropogenic impacts before it was established as a protection area, with farms, the presence of people and vehicles, hunting, logging, and the introduction of exotic species (Melo 2017). Despite changes over the years, endemic species can still be found growing in the TES; its vegetation is currently composed of secondary forest regenerating for over 30 years, and harbors approximately 41 families of herbs, shrubs, and trees (Melo 2017). A large portion of the station's territory was closed to the public in 2006 to guarantee its conservation (Melo 2017). Numerous phytosociological (Andrade \& Rodal 2004, Moura et al. 2012) and scientific studies have been undertaken in the TES (Andrade \& Rodal 2004, Júnior 2005, El-Deir et al. 2012, Andrade \& Câmara 2008, Melo 2017, among others) and have demonstrated its wide biodiversity, with the station contributing greatly to the conservation of the local biota.

Our basic knowledge of the plant diversity at TES is not fully sufficient to guarantee its conservation, however, as there is still a general lack of information concerning the geographic distributions of its species, their conservation statuses, and records of new occurrences. We therefore undertook a detailed survey of the Angiosperms in the TES forest, to gather additional data concerning its species composition.

\section{Materials and Methods}

The present study was carried out in a remnant of seasonal semideciduous lowland forest at the Tapacurá Ecological Station (TES), located in the municipality of São Lourenço da Mata (-8.004, -35.111), in Pernambuco State, Brazil (Figure 1). The regional climate is hot and humid (type As' by the Köppen classification), with an average annual precipitation rate of 1,900 $\mathrm{mm}$ (BENTO et al. 2018). The geomorphology there is characterized by remobilized surfaces and a landscape of low and wide hills, with rocks of the Precambrian crystalline basement and sediments of the Cabo Formation (conglomerates of Aptiano clay) and alkaline vulcanites of the Ipojuca Formation, with elevations varying from 140 to 200 masl (CPRH 2017).

The vegetation in the TES is a seasonal semideciduous lowland forest according to the phytogeographic classification of IBGE (2012). The station covers a total area of approximately 800 ha, with a predominance of Atlantic Forest surrounded by dry forest, "capoeiras", and anthropized areas of cottages or small farms that cultivate several commercial and/or exotic species such as jambolão (Syzygium sp.), palm (Elaeis guineenses L.), papaya (Carica sp.), coconut (Cocos nucifera L.), mango (Mangifera indica L.), and bananas (Musa sp.). Atlantic Forest vegetation covers approximately 400 ha, distributed in three fragments: Mata do Alto da Buchada, Mata do Camucim, and Mata do Toró; an additional 400 ha corresponds to the Tapacurá Lake - resulting from the damning the Tapacurá River, an affluent of the Capibaribe River (Andrade et al. 2009).

Collections of flowering or fruiting species were undertaken in September/2019 and January/2021, during the wet and dry seasons (Figure 1), focusing on the three forest fragments mentioned above, following established trails but also exploring other less-accessible areas in different sites within the TES. The botanical material was collected and herborized employing standard taxonomic methodologies (Fidalgo \& Bononi 1989). Plant identifications were established through the use of identification keys, comparisons with type collections, consulting the specialized literature and specialists of specific taxa, and comparisons with PEUFR herbarium collections. Additionally, the SpeciesLink database (http://splink.cria.org.br/) as well as specimens deposited in the collections of the HST, IPA, PEUFR and UFP herbaria (acronyms according to Thiers 2020). For distribution, We consulted the labels of exsiccates, Flora of Brazil (2020) website, among floristic studies undertaken in northeastern Brazil and/or in Pernambuco State (Souza \& Wanderley 2000, Sobral-Leite et al. 2010, Hatori 2009, Santos et al. 2013, Soares Neto 2014). The species occurrences were recorded in the TES database to create a species list as presented here. The floristic list classifications are based on the Angiosperm Phylogeny Group (APG IV 2016).

\section{Results}

A total of 479 species were recorded, belonging to 81 families and 285 genera, some of their representatives are showed in the figures 2, 3 and 4 (Table 1 and Figure 5). Of those, 71.60\% were Eudicotyledons (58 spp.), $22.22 \%$ were monocotyledons ( $18 \mathrm{spp}$.), and $4.94 \%$ belong to the magnoliidae group (4 spp.; Table 1). The families were: Fabaceae (68 spp.), Rubiaceae (25), Malvaceae (20), Myrtaceae (20), Cyperaceae (19), Poaceae (19), Bromeliaceae (18), Euphorbiaceae (17), Asteraceae (16), and Sapindaceae (14). Those ten families represented $49.27 \%$ of all of the species identified in the study area (Figure 6). The results also confirmed 44 new occurrences for Pernambuco State.

Twenty-six families were represented by only a single species: Alismataceae, Alstroemeriaceae, Apiaceae, Araliaceae, Begoniaceae, Burseraceae, Cactaceae, Cannabaceae, Caryophyllaceae, Costaceae, Dioscoreaceae, Gesneriaceae, Heliconiaceae, Hernandiaceae, Hypericaceae, Iridaceae, Linderniaceae, Marcgraviaceae, Menispermaceae, Olacaceae, Phytolaccaceae, Piperaceae, Podostemaceae, Polygalaceae, Smilacaceae, and Violaceae. 
Angiosperm in Tapacurá

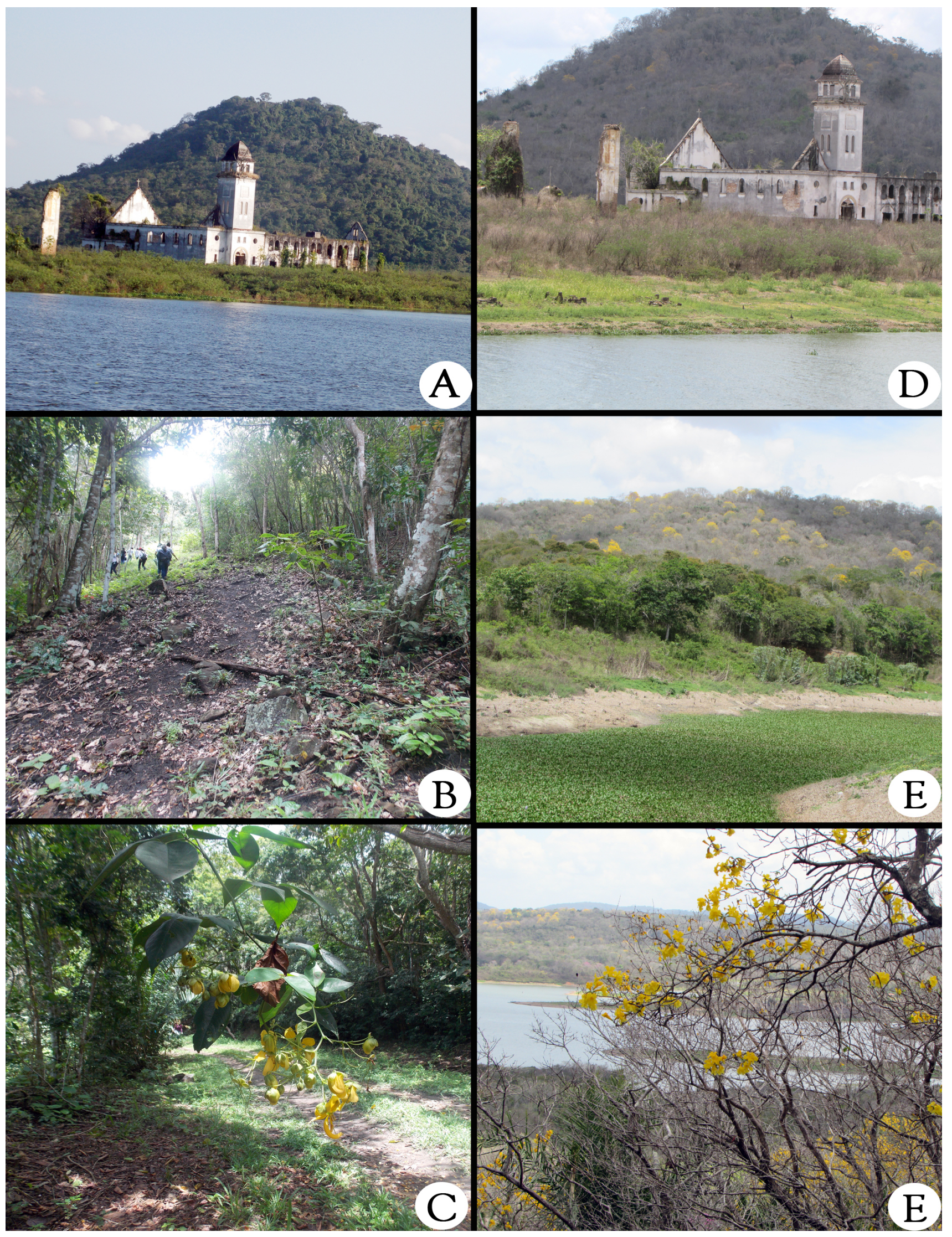

Figure 1. A-C. TSE during the wet season; D-F. TSE during the dry season. 
Pereira-Silva, RA. et al.
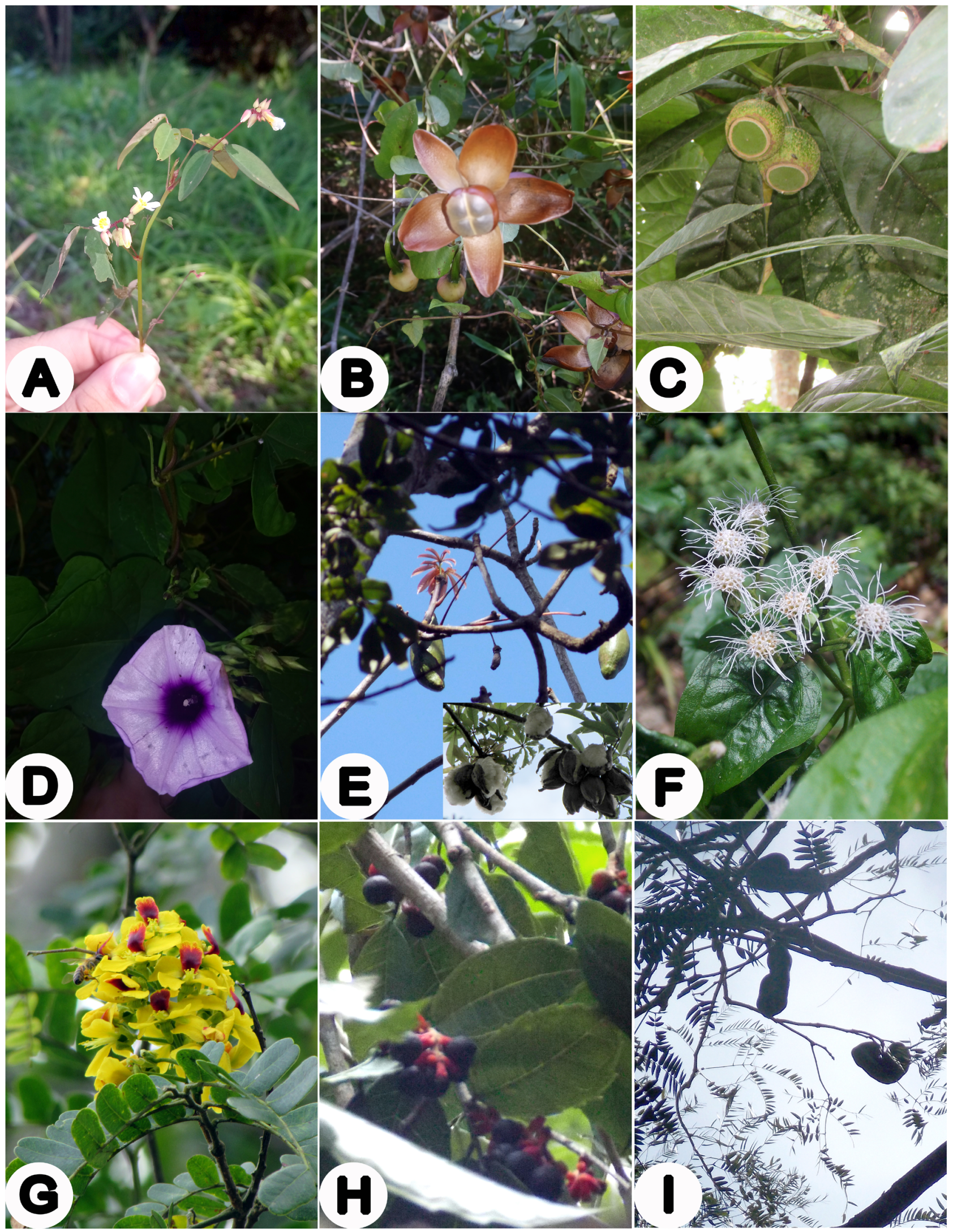

Figure 2. A. Oxalis physocalyx Zucc. Ex Progel.; B.Merremia cf. tuberosa Rendle; C. Gustavia augusta L.; D. Ipomoea marcellia Meisn.; E. Pseudobombax marginatum (A. St. Hil.Juss \& Cambess.) A. Robyns.; F. Chromolaena odorata (L.) R.M.King \& H.Rob.;G. Paubrasilia echinata (Lam.) Gagnon, H, C. Lima \& G. P. Lewis; H. Sorocea bonplandii W.C.Burger, Lanj. \& Wess.Boer; I. Enterolobium contortisiliquum (Vell.) Morong. 


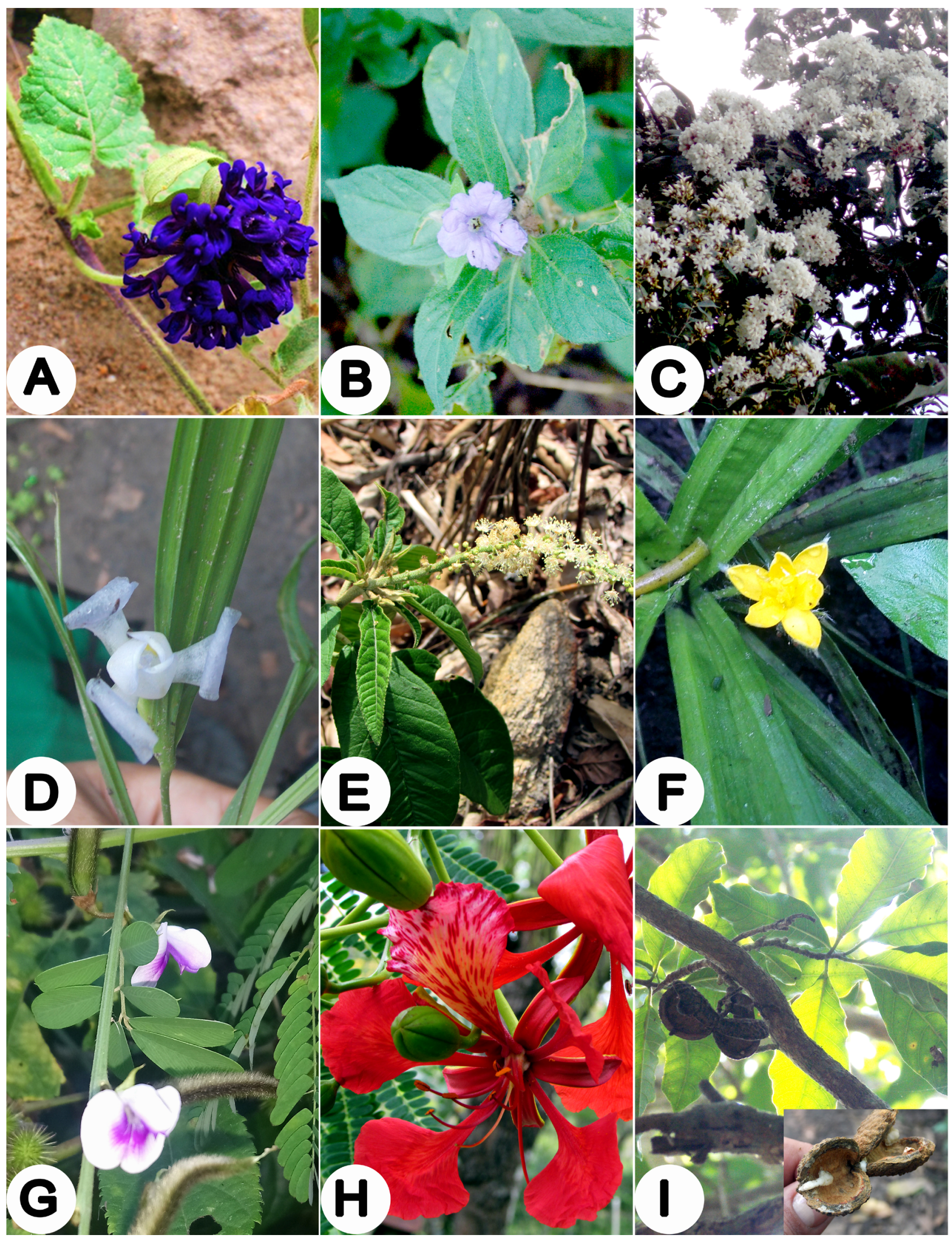

Figure 3. A. Rhaphiodon echinus (Nees\& Mart.) Schauer; B. Ruellia grandiflora Poir; C. Cordia goeldiana Huber; D. Cipura paludosa Aubl. E. Croton heliotropiifolius Kunth.;F Hipoxis hirsuta G. Tephrosia noctiflora; H. Delonix regia (Hook.) Raf.; I. Tabernaemontana hystrix Steud. 


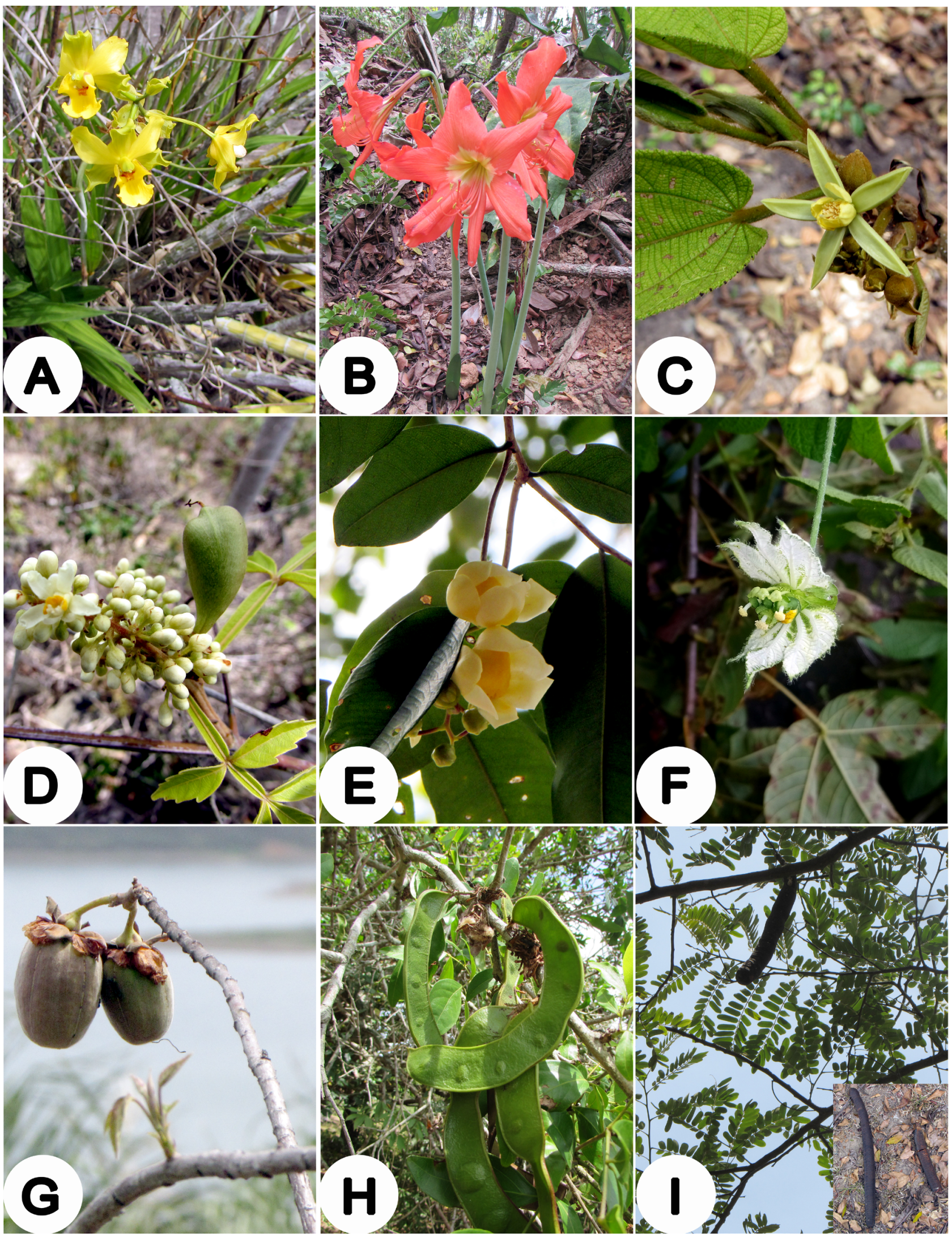

Figure 4. Cyrtopodium cardiochilum Lindl.; B. Hippeastrum puniceum (Lam.) Voss; C. Apeiba tibourbou Aubl. D. Paullinia sp L..; E. Eschweilera ovata Mart. ex Miers; F. Dalechampia pernambucensis Baill. Spreng; G. Cochlospermum vitifolium (Willd.) Spreng; H. Adenanthera pavonina L. I. Cassia grandis L. f. 
Table 1. Angiosperms of the TSE. $H=$ Herb; $S U=$ Subshrub; $S=S h r u b ; T=$ Tree; $V=$ Vine.

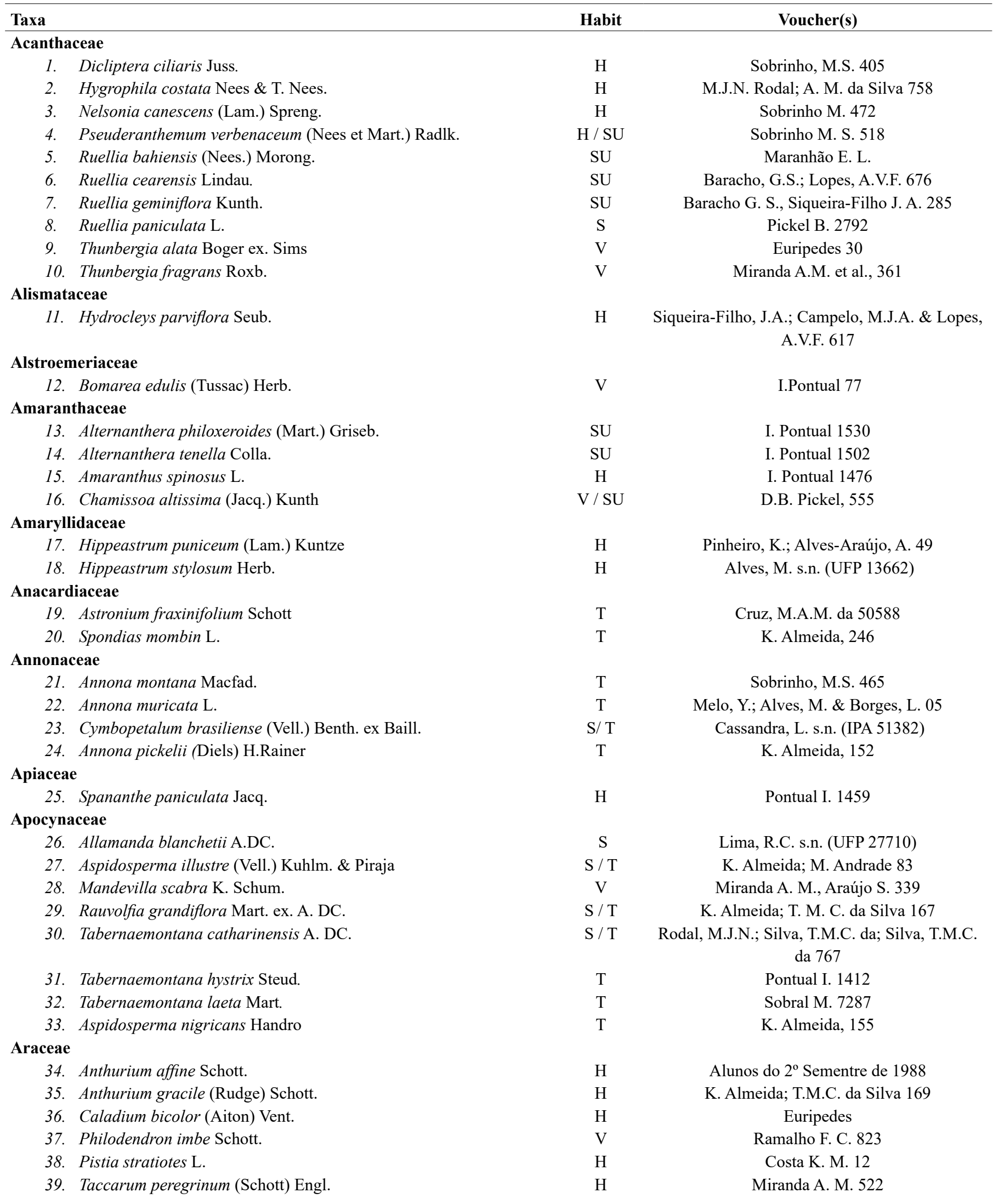




\section{Arecaceae}

40. Bactris pickelii Burret.

41. Desmoncus polyacanthos Mart..

Araliaceae

42. Hydrocotyle ranunculoides L.F.

Asteraceae

43. Delilia biflora (L.) Kuntze

44. Mikania micrantha Kunth

45. Acanthospermum hispidum DC.

46. Bidens pilosa $\mathrm{L}$.

47. Centratherum punctatum Cass.

48. Eclipta prostrata (L.) L.

49. Emilia fosbergii Nicolson.

50. Emilia sonchifolia DC.

51. Lactuca sativa $\mathrm{L}$.

52. Pterocaulon alopecuroides (Lam.) DC.

53. Mikania cordifolia (L. f.) Willd.

54. Sphagneticola trilobata (L.) Pruski

55. Synedrella nodiflora (L.) Gaertn.

56. Verbesina macrophylla (Cass.) S. F. Blake.

57. Vernonanthura brasiliana (L.) H. Rob.

58. Wedelia villosa Gardner

Begoniaceae

59. Begonia reniformis Dryand.

Bignoniaceae

60. Adenocalymma divaricatum Miers.

61. Adenocalymma comosum (Cham.) DC.

62. Adenocalymma hypostictum Bureau \& K. Schum.

63. Amphilophium crucigerum (L.) L. Lohmann.

64. Handroanthus serratifolius (Vahl) S.O. Grose.

65. Lundia longa (Vell.) D.C.

66. Adenocalymma coriaceum A. DC.

67. Bignonia binata Thunb.

Boraginaceae

68. Cordia sellowiana Cham.

69. Cordia superba Cham

70. Cordia taguahyensis Vell.

71. Cordia toqueve Aubl.

72. Cordia trichotoma (Vell.) Arráb. ex Steud.

73. Heliotropium elongatum (Lehm.) I.M.Johnst.

74. Heliotropium angiospermum Murray.

75. Varronia polycephala Lam.

\section{Bromeliaceae}

76. Aechmea mertensii (G.Mey.) Schult. \& Schult.f.

77. Aechmea fulgens Brongn.

78. Aechmea leptantha (Harms) Leme \& J.A. Siqueira

79. Aechmea lingulata (L.) Baker.

80. Aechmea muricata (Arruda) L.B. Sm.

81. Aechmea tomentosa Mez.

82. Billbergia morelii Brongn.

83. Bromelia karatas L.

84. Cryptanthus fosterianus L.B. Sm.
$\mathrm{P}$

$\mathrm{H} / \mathrm{V}$

$\mathrm{H}$

$\mathrm{H}$

V

$\mathrm{H}$

$\mathrm{H}$

SU

$\mathrm{H}$

$\mathrm{H}$

$\mathrm{H}$

$\mathrm{H}$

SU

V

$\mathrm{H}$

$\mathrm{H} / \mathrm{S}$

$\mathrm{S}$

$\mathrm{S}$

$\mathrm{S}$

SU

V

V

$\mathrm{S} / \mathrm{V}$

$\mathrm{V}$

$\mathrm{T}$

V

V

$\mathrm{V}$

$\mathrm{T}$

$\mathrm{S} / \mathrm{T}$

$\mathrm{S} / \mathrm{T}$

$T$

$\mathrm{T}$

$\mathrm{H} / \mathrm{SU}$

$\mathrm{H} / \mathrm{SU}$

$\mathrm{S} / \mathrm{SU}$
A.M. Miranda; E. Araújo, S.I. Silva et G. Gamarras 3610

Santos, E.G. dos; Silva, A.M.; Silva, A.M. s.n. (IPA 92931)

Sobrinho, M.S. 309

Santos, E.G. dos; Silva, A.M.; Silva, A.M. s.n. (IPA 92935)

K. Almeida; A. Lima 199

Lopes A. V. F. s.n. E.S. Silva, 19

V. Sobrinho s.n. (K 000977498)

M.J.N. Rodal; T.M.C. da Silva 770

Lopes, A.V.F. s.n.

R. Barreto; R. Lima S/N

I. Pontual 1302

K. Almeida 156

I. Pontual 77

Baracho, G.S.; Siqueira-Filho, J.A. 533

K. Almeida, 126

Andrade-Lima 63-4186

I. Pontual 1312

Lopes, A.V.F. s.n.

Sousa, G.M.; Wanderley, M.G.L. 165

Sousa, G.M. 169

Sousa, G.M.; Wanderley, M.G.L. 164

Lopes, A.V.F. s.n.

Siqueira-Filho, J.A. 612

I. Pontual 1537 


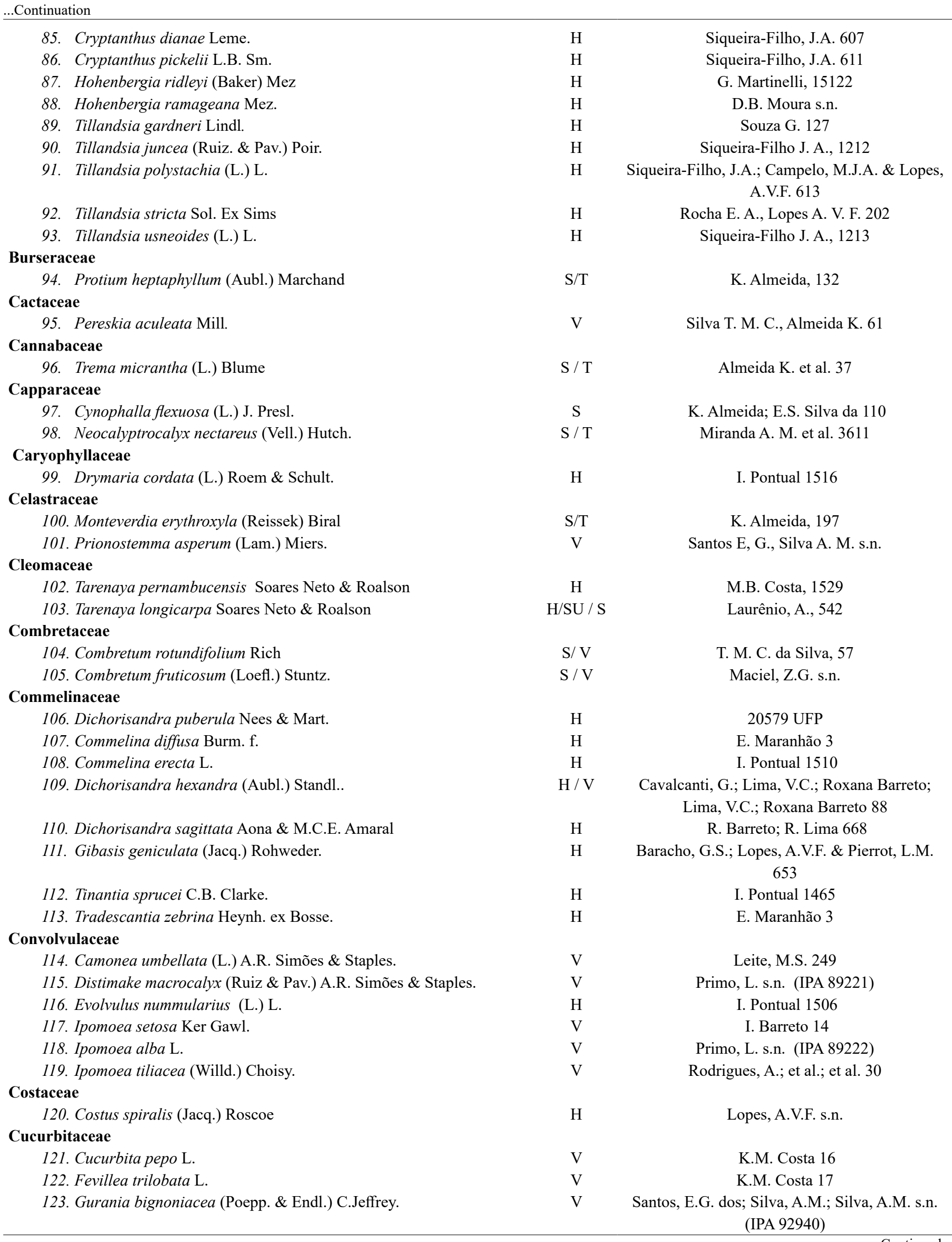




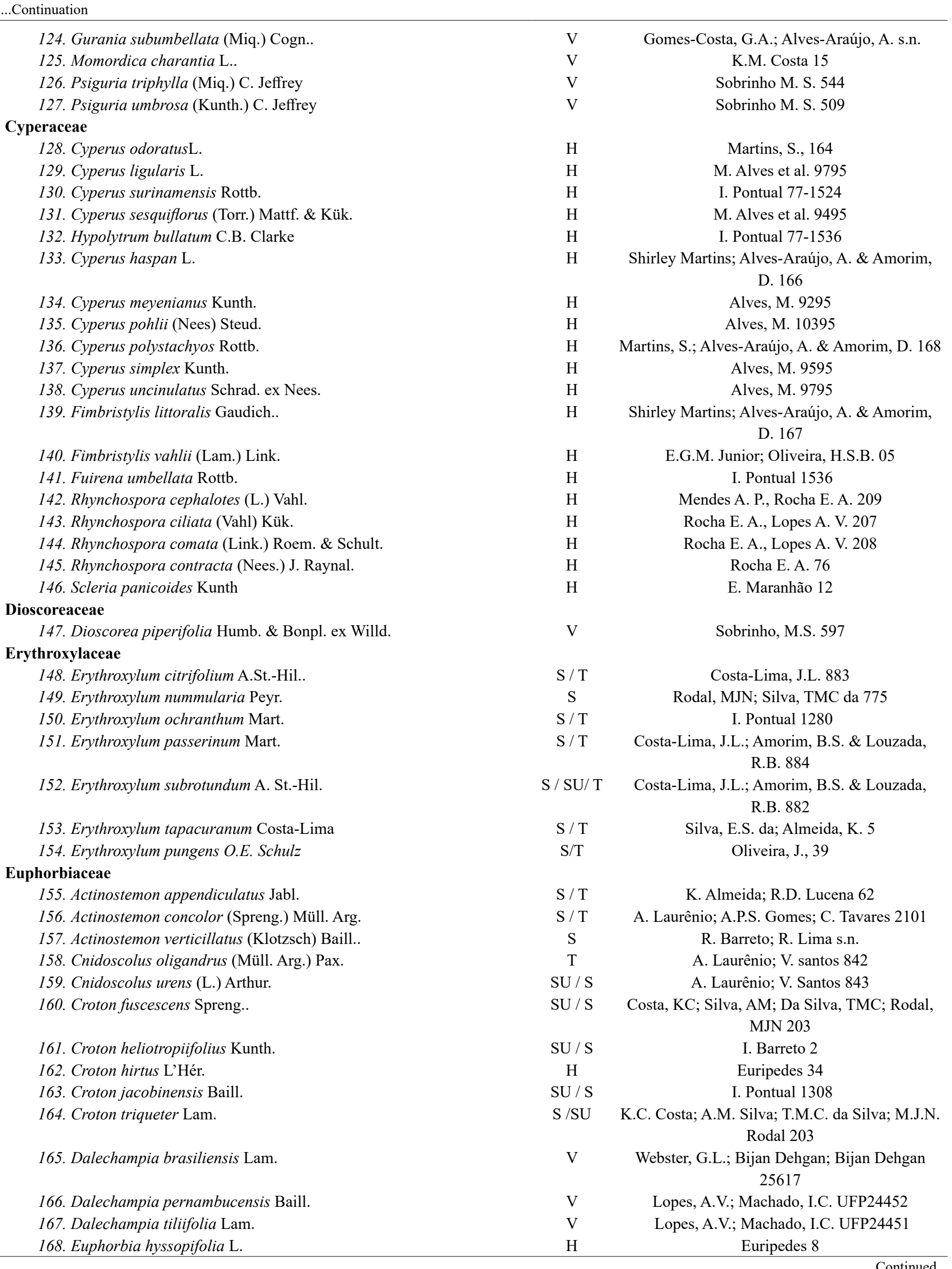




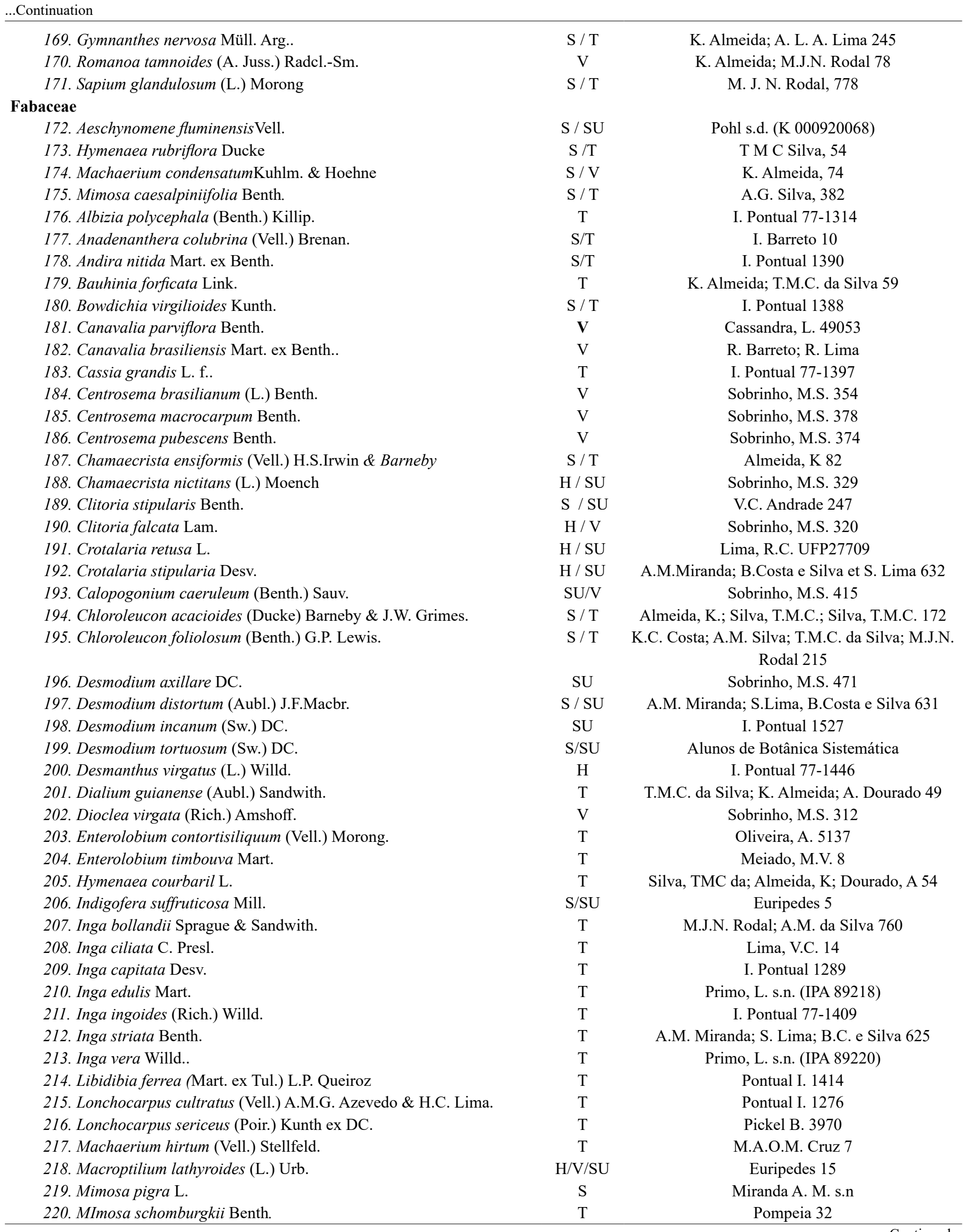


221. Mimosa sensitiva L.

222. Mucuna sloanei Fawcett. \& Rendle.

223. Paubrasilia echinata (Lam.) Gagnon, H.C. Lima \& G.P. Lewis

224. Plathymenia reticulata Benth.

225. Pterocarpus rohrii Vahl.

226. Rhynchosia phaseoloides (SW.) DC.

227. Samanea saman (Jacq.) Merr..

228. Samanea tubulosa (Benth.) Barneby \& J. W. Grimes

229. Schnella outimouta (Aubl.) Wunderlin.

230. Schnella splendens (Kunth.) Benth.

231. Senegalia tenuifolia (L.) Britton. \& Rose.

232. Senna alata (L.) Roxb.

233. Senna georgica H. S. Irwin. \& Barneby.

234. Senna macranthera (DC. ex Collad.) H. S. Irwin \& Barneby.

235. Stylosanthes scabra Vogel.

236. Swartzia pickelli Killip. ex. Ducke.

237. Tephrosia cinerea (L.) Pers

238. Zornia latifolia Sm.

239. Zygia latifolia (L.) Fawc. \& Rendle.

\section{Gentianaceae}

240. Schultesia guianensis (Aubl.) Malme.

241. Voyria flavescens Griseb.

\section{Gesneriaceae}

242. Drymonia serrulata (Jacq.) Mart.

\section{Heliconiaceae}

243. Heliconia psittacorum L.F.

\section{Hernandiaceae}

244. Sparattanthelium botocudorum Mart.

\section{Hypericaceae}

245. Vismia guianensis (Aubl.) Choisy.

Iridaceae

246. Cipura paludosa Aubl.

\section{Lamiaceae}

247. Aegiphila integrifolia (Jacq.) Moldenke

248. Aegiphila pernambucensis Moldenke

249. Aegiphila racemosa Vell.

250. Aegiphila verticillata Vell.

251. Ocimum gratissimum L.

252. Ocimum campechianum Mill.

253. Mesosphaerum pectinatum (L.) Kuntze

254. Leonotis nepetifolia (L.) R.Br.

255. Vitex polygama Cham.

256. Vitex rufescens A. Juss.

\section{Lauraceae}

257. Ocotea canaliculata (Rich.) Mez

258. Ocotea glauca (Nees \& Mart.) Mez

259. Ocotea fasciculata (Nees) Mez

\section{Lecythidaceae}

260. Cariniana legalis (Mart.) Kuntze.

261. Eschweilera alvimii S.A. Mori.

262. Eschweilera ovata (Cambess.) Miers.

\section{$\mathrm{S} / \mathrm{SU}$ \\ $\mathrm{V}$ \\ $\mathrm{T}$ \\ $\mathrm{T}$ \\ $\mathrm{T}$ \\ V \\ $\mathrm{T}$ \\ $\mathrm{T}$ \\ $\mathrm{V} / \mathrm{S}$ \\ $\mathrm{V}$ \\ $\mathrm{S} / \mathrm{V}$ \\ $\mathrm{SU} / \mathrm{S} / \mathrm{T}$ \\ $\mathrm{SU} / \mathrm{S} / \mathrm{T}$ \\ $\mathrm{S} / \mathrm{T}$ \\ $\mathrm{H} / \mathrm{SU}$ \\ $\mathrm{T}$ \\ $\mathrm{S} / \mathrm{SU}$ \\ $\mathrm{SU}$ \\ $\mathrm{S} / \mathrm{T}$}

$\mathrm{H}$

$\mathrm{H}$

V

$\mathrm{H}$

S

$\mathrm{S} / \mathrm{T}$

$\mathrm{H}$

$\mathrm{S} / \mathrm{T}$

$\mathrm{S} / \mathrm{T}$

$\mathrm{S} / \mathrm{V}$

$\mathrm{SU} / \mathrm{S} / \mathrm{T}$

$\mathrm{SU} / \mathrm{S}$

$\mathrm{H} / \mathrm{S} / \mathrm{SU}$

$\mathrm{H} / \mathrm{S} / \mathrm{SU}$

$\mathrm{H}$

$\mathrm{S} / \mathrm{T}$

$\mathrm{T}$

$\mathrm{T}$

$\mathrm{T}$

$\mathrm{S} / \mathrm{T}$
I. Pontual 1443

Baracho, G.S.; Lopes, A.V.F. 677

Pontual I. 1389

Almeida K., Silva T. M. C. 142

Pontual I. 1404

Sobrinho M. S. 407

K. Almeida; E. Santos 1

Rita Pereira et al. 54704

Santos E. G., Silva A. M., s.n.

Esteves F. s.n.

Pontual I. 1454

Coelho L. 50645

Miranda A. M. et al. 3604

Sobrinho M. S. 428

Pontual I. 1497

Lima R., Barreto R. s. n.

Sobrinho M. 571

Sobrinho M. S. 327

M.J.N. Rodal; A.M. da Silva 766

Miranda A. M., et al. 620

Melo A. et al., 443

Baracho, G.S.; Siqueira, J.A. \& Lopes, A.V.F. 627

Gomes, V. s.n.

E.S. da Silva; K. Almeida 8

Silva, TMC; Almeida, K 40

Alunos de Botânica Sistemática 1988

K. Almeida, 3

Sobrinho, M. 542

A.M. Miranda; E.Araújo, S.I.Silva et G.Gamarras 3616

Almeida, K.; Santos, E.; Santos, E. 3

Albuquerque 74

Albuquerque 72

Alunos $2^{\circ}$ Semestre s.n. (PEUFR 11998)

Euripedes 19

Costa K. C. et al., 210

Almeida K., Santos E. 2

Santos S. O. 319

E. S. Silva, 2

R. Barreto 8

R. M. Silva 1

R. M. Silva 2

I. Pontual 1403 
263. Gustavia augusta L.

264. Lecythis pisonis Cambess.

\section{Linderniaceae}

265. Lindernia crustacea F. Muell.

\section{Loganiaceae}

266. Strychnos bahiensis Krukoff \& Barneby

267. Spigelia flemmingiana Cham. \& Schltdl.

\section{Malpighiaceae}

268. Amorimia septentrionalis W.R.Anderson

269. Bunchosia maritima (Vell.) J.F.Macbr.

270. Malpighia glabra L.

271. Stigmaphyllon puberulum Griseb.

272. Tetrapterys mucronata Cav.

273. Byrsonima sericea DC.

Malvaceae

274. Apeiba tibourbou Aubl.

275. Guazuma ulmifolia Lam.

276. Luehea ochrophylla Mart.

277. Luehea paniculata Mart. \& Zucc.

278. Malachra radiata $\mathrm{L}$.

279. Sida glomerata Cav.

280. Sidastrum micranthum (A. St.-Hil.) Fryxell

281. Sidastrum quinquenervium (Duchass. ex Triana \& Planch.) Fryxell

282. Sida urens L.

283. Urena lobata $\mathrm{L}$.

284. Wissadula periplocifolia (L.) C. Presl ex Thwaites

285. Sida rhombifolia L.

286. Sida spinosa L.

287. Abutilon ramiflorum A.St.-Hil.

288. Christiana africana DC.

289. Helicteres macropetala A.St.-Hil.

290. Helicteres ovata Lam.

291. Sida jussiaeana DC.

292. Triumfetta semitriloba Jacq.

293. Wissadula amplissima (L.) R.E.Fr.

\section{Marantaceae}

294. Maranta leuconeura E. Morren

295. Hylaeanthe hexantha (Poepp. \& Endl.) A.M.E. Jonker \& Jonker

296. Ctenanthe compressa (A.Dietr.) Eichler.

297. Goeppertia effusa Saka \& Lombardi.

298. Goeppertia cylindrica (Roscoe) Borchs. \& S. Suárez.

299. Goeppertia villosa (Lindl.) Borchs. \& S. Suárez.

300. Maranta divaricata Roscoe

301. Maranta protracta Miq.

302. Stromanthe porteana Griseb.

303. Stromanthe tonckat (Aubl.) Eichler

\section{Marcgraviaceae}

304. Schwartzia brasiliensis (Choisy) Bedell ex Gir--Cañas

\section{Melastomataceae}

305. Clidemia hirta (L.) D. Don.

306. Clidemia capitellata (Bonpl.) D.Don..

307. Miconia albicans (SW) Triana

308. Miconia minutiflora (Bonpl.) DC.
$\mathrm{S} / \mathrm{T}$

$\mathrm{T}$

$\mathrm{H}$

V

$\mathrm{H}$

V

$\mathrm{S} / \mathrm{T}$

$\mathrm{T}$

V

$\mathrm{V}$

$\mathrm{S} / \mathrm{T}$

$\mathrm{T}$

$\mathrm{T}$

$\mathrm{T}$

$\mathrm{S}$

$\mathrm{H}$

$\mathrm{SU} / \mathrm{S}$

$\mathrm{S} / \mathrm{SU}$

SU

SU

$\mathrm{S} / \mathrm{SU}$

$\mathrm{SU} / \mathrm{S}$

$\mathrm{H}$

$\mathrm{SU}$

$\mathrm{S}$

$\mathrm{T}$

$\mathrm{SU} / \mathrm{S} / \mathrm{T}$

$\mathrm{S}$

SU

SU

S / SU

$\mathrm{H}$

$\mathrm{H}$

$\mathrm{H}$

$\mathrm{H}$

$\mathrm{H}$

$\mathrm{H}$

$\mathrm{H}$

$\mathrm{H}$

$\mathrm{H}$

$\mathrm{H}$

V

$\mathrm{S}$

$\mathrm{S}$

$\mathrm{S} / \mathrm{T}$

$\mathrm{S} / \mathrm{T}$
I. Pontual 1424

I. Pontual 1402

Pontual I. 1483

K. Almeida 130

Chiappeta A. 5438

Miranda, AM; Araújo, E; Silva, SI; Gamarras, G 3606

Sobrinho, M.S. 507

Melo Y. et al. 4

Pickel B. 3260

Sobrinho M. 582

K. Almeida, 95

V.C. Andrade 237

Lima, V.C. 12

Miranda A. M. et al. 3596

Almeida K. Santos E. 12

Gallindo F. et al. CFPE-648

Baracho G. S., Siqueira-Filho J. A. 282

Baracho G. S., Siqueira-Filho J. A. 531

Sobrinho, M. 584

Barreto I. 26

Sobrinho M. S. 570

Miranda A. M. 521

I. Pontual 77-1471

G. S. Baracho 455, 456

B.S. Amorim, 689

K. Almeida, 183

Batista, E. s.n. (UFRN 9954)

G. S. Baracho, 286

G.S. Baracho, 291

Amorim, B.S., 676

G.S. Baracho, 288

Alves et al. 12-95

N.K. Luna, 405

Arns, K.N.Y. 138

A.M. Miranda; et al. 617

Baracho, G.; Lopes, A.V.F.; Pierrot, L.M.; Lopes,

A.V.F.; Pierrot, L.M. 491

Yoshida, A. 681

Luna N. K. et al. 460

Luna N. K. 459

Alves M. et al., 33794

Alves M. et al., 9695

Ariadna V F Lopes, 21785

$M^{a}$. Rita Sales s.n.

Almeida, K. 103

Sobrinho M. S. 510

Silva T. M. C. 10 
309. Miconia navioensis Wurdack.

310. Miconia prasina (Sw.) DC.

311. Miconia serialis DC.

312. Pterolepis trichotoma (Rottb.) Cogn.

313. Clidemia biserrata DC.

\section{Meliaceae}

314. Trichilia hirta L.

315. Guarea guidonia (L.) Sleumer.

Menispermaceae

316. Cissampelos glaberrima A.St.-Hil..

Moraceae

317. Dorstenia pernambucana Arruda.

318. Sorocea hilarii Gaudich.

Myrtaceae

319. Calyptranthes lucida Mart. ex DC.

320. Campomanesia eugenioides (Cambess.) D.Legrand ex Landrum

321. Myrcia inaequiloba (DC.) Legrand

322. Myrcia felisbertii (DC.) O. Berg

323. Eugenia luschnathiana (O. Berg) Klotzsch ex B.D.Jacks.

324. Psidium oligospermum Mart. ex DC.

325. Campomanesia aromatica (Aubl.) Griseb.

326. Campomanesia dichotoma (O. Berg) Mattos.

327. Eugenia astringens Cambess.

328. Eugenia candolleana DC.

329. Eugenia duarteana Cambess.

330. Eugenia gaudichaudiana O.Berg.

331. Eugenia hirta O. Berg.

332. Eugenia umbrosa O. Berg.

333. Myrcia guianensis (Aubl.) DC.

334. Myrcia insularis Gardner

335. Myrcia tomentosa (Aubl.) DC.

336. Myrciaria ferruginea O.Berg.

337. Myrciaria glazioviana (Kiaersk.) G.M.Barroso ex Sobral

338. Psidium guineense $\mathrm{Sw}$

Nyctaginaceae

339. Guapira laxa (Netto) Furlan.

340. Guapira hirsuta (Choisy) Lundell.

341. Guapira nitida (Schimdt) Lundell

\section{Nymphaeaceae}

342. Nymphaea lasiophylla Mart. \& Zucc.

343. Nymphaea rudgeana G. Mey

\section{Olacaceae}

344. Ximenia americana L.

Onagraceae

345. Ludwigia helminthorrhiza Mart. H. Hara

346. Ludwigia hyssopifolia (G. Don.) Exell

347. Ludwigia peploides (Kunth.) P. H. Raven.

\section{Orchidaceae}

348. Vanilla chamissonis Klotz.

349. Campylocentrum fasciola (Lindl.) Cogn.

350. Gomesa barbata (Lindl.) Chase.
$\mathrm{S}$

Almeida K. 8

Almeida K. 98

M.J.N. Rodal; A.M. da Silva 748

Sobrinho M. S. 595

K. Almeida, 103

Miranda A. M. et al. 517

Oliveira, A. s.n. (IPA 51371 )

Sobrinho, M.S. 502

Sobrinho, M.S. 562

Almeida, K.; Andrade, M.; Andrade, M. 84

K. Almeida, 73

K. Almeida, 129

K. Almeida, 94

K. Almeida, 31

I. Pontual 77-1416

I. Pontual 77-1286

Amorim, BS; Costa-Lima, JL; Louzada, RB 1788

I. Pontual 1400

Almeida, K; Silva, TMC 120

K.C. Costa; A.M. da Silva; T.M.C. da Silva;

M.J.N. Rodal 207

I. Pontual 1294

M.Sobral 7296

E.S. da Silva; K. Almeida 1

E.S. da Silva; Antônio 29

Sobral M. 7290

Almeida, K.; Santos, E.; Santos, E. 17

A. Laurênio; V. Santos 844

K. Almeida; R.D. Lucena 66

Amorim B. S. et al. 687

Almeida K. 51

Sobrinho, M.S. 458

SU /S / T Silva, T.M.C. da; Almeida, K.; Almeida, K. 68

T Rodal, M.J.N.; Silva, A.M. da; Silva, A.M. da 749

Pickel B. 3725

Pickel B. 2343

Sobrinho M. S. 443

Pontual I. 77-1534

Sobrinho M. 592

Leite M. S. 246

A. L. A. Lima, 9

Moraes s.n. (EAN, HB)

Vilaça, M.; Nepomuceno, A.; Guedes, F.M. \& Cavalcanti, D. 102 
351. Oeceoclades maculata Lind1.

352. Polystachya concreta (Jacq.) Garay \& H. R. Sweet

353. Rodriguezia bahiensis Rchb.f.

354. Sacoila lanceolata (Aubl.) Garay

355. Sarcoglottis acaulis (Sm.) Schltr.

356. Maxillaria subrepens (Rolfe) Schuit. \& M.W.Chase

357. Vanilla planifolia Jacks. ex Andrews

\section{Oxalidaceae}

358. Oxalis cratensis Oliver.

359. Oxalis psoraleoides Kunth

Phylantaceae

360. Phyllanthus acuminatus Vahl.

361. Phyllanthus juglandifolius Willd.

Phytolaccaceae

362. Rivina humilis $\mathrm{L}$.

\section{Piperaceae}

363. Piper marginatum Jaqc.

Plantaginaceae

364. Achetaria erecta (Spr.) Wettst.

365. Stemodia foliosa Benth.

366. Scoparia dulcis L.

\section{Poaceae}

367. Axonopus scoparius (Flüggé) Kuhlm.

368. Andropogon bicornis Forssk.

369. Cenchrus echinatus L.

370. Cynodon dactylon (L.) Pers.

371. Eleusine indica (L.) Gaertn.

372. Hyparrhenia rufa (Nees) Stapf.

373. Lasiacis divaricata (L.) Hitchc.

374. Lasiacis ligulata Hitchc. \& Chase.

375. Lasiacis sorghoidea (Desv.) Hitchc. \& M.A. Chase.

376. Paspalum coryphaeum Trin.

377. Paspalum molle Poir.

378. Paspalum paniculatum L.

379. Paspalum plicatulum Michx.

380. Paspalum virgatum $L$.

381. Raddia brasiliensis Bertol.

382. Setaria globulifera (Steud.) Griseb.

383. Setaria scabrifolia (Nees) Kunth.

384. Sporobolus indicus (L.) R. Br.

385. Tragus berteronianus Schult.

Podostemaceae

386. Tristicha trifaria (Bory ex Willd.) Spreng.

Polygalaceae

387. Securidaca lanceolata St.-Hil.

\section{Polygonaceae}

388. Coccoloba glaziovii Lindau

389. Coccoloba alnifolia Casar.

390. Coccoloba mollis Casar.

391. Coccoloba parimensis Benth.

392. Polygonum ferrugineum Wedd.

393. Polygonum punctatum Elliott.

394. Triplaris gardneriana Wedd.
$\mathrm{H}$

$\mathrm{H}$

$\mathrm{H}$

$\mathrm{H}$

$\mathrm{H}$

$\mathrm{H}$

$\mathrm{H}$

$\mathrm{H}$

$\mathrm{S} / \mathrm{SU}$

$\mathrm{S} / \mathrm{T}$

$\mathrm{S} / \mathrm{T}$

$\mathrm{H}$

$\mathrm{S}$

$\mathrm{H} / \mathrm{SU}$

$\mathrm{H} / \mathrm{SU} / \mathrm{S}$

$\mathrm{H} / \mathrm{SU}$

$\mathrm{H}$

$\mathrm{H}$

$\mathrm{H}$

$\mathrm{H}$

$\mathrm{H}$

$\mathrm{H}$

$\mathrm{H} / \mathrm{V} / \mathrm{SU}$

$\mathrm{H}$

$\mathrm{H}$

$\mathrm{H}$

$\mathrm{H}$

$\mathrm{H}$

$\mathrm{H}$

$\mathrm{H}$

$\mathrm{H}$

$\mathrm{H}$

$\mathrm{H}$

$\mathrm{H}$

$\mathrm{H}$

$\mathrm{H}$

V

$\mathrm{S} / \mathrm{T}$

$\mathrm{S} / \mathrm{T}$

$\mathrm{T}$

$\mathrm{S} / \mathrm{V}$

$\mathrm{H}$

$\mathrm{H}$
Sobrinho M. S. 560

Alves M. 10095

Lima R. C. s.n.

Lopes A. V. F. s.n.

Vilaça M. et al., 105

Siqueira-Filho J. A. 1214

Pessoa E. et al., 403

Nádia T. L. 207

T. M. C. da Silva, 28

Sobrinho M. S. 551

Silva, T.M.C. da; Almeida, K.; Almeida, K. $60 \mathrm{H}$

Pickel B. 1871

Silva E. S., Almeida K. 16

Sobrinho, M.S. 610

Sobral M. 7286

Pontual I. 1488

Euripedes 20

I. Pontual 1548

I. Pontual 1467

I. Pontual 1442

Euripedes

Euripedes 28

Melo J. F. 11734

Pontual I. 1540

Alves M. UFP 10795

Pickel B. 1561

Maciel J. R. 172

Maciel J. R. 167

Maciel J. R. 170

Maciel J. R. 168

C. A. Silva s.n.

C. A. Silva s.n.

C. A. Silva s.n.

I. Pontual 1498

Alves M. 10495

Pickel B. 1420

Chiappeta A. 408

Almeida, K., 102

Sobrinho, M.S. 375

Rodal, M.J.N.; Silva, A.M. da; Silva, A.M. da 750

Almeida, K. 102

Costa K. M. 10

Costa K. M. 18

Cirne E. s.n. 


\section{Pontederiaceae}

395. Eichhornia crassipes (Mart.) Solms.

396. Heteranthera oblongifolia Mart.

Potamogetonaceae

397. Potamogeton polygonus Cham. \& Schltdl.

398. Potamogeton pusillus L.

Rhamnaceae

399. Gouania blanchetiana Miq.

400. Colubrina glandulosa Perkins.

401. Gouania colurnifolia Reissek.

402. Gouania lupuloides (L.) Urb.

\section{Rubiaceae}

403. Chomelia occidentalis Müll.Arg.

404. Alseis floribunda Schott.

405. Alseis pickelii Pilger \& Schmale.

406. Alseis latifolia Gleason.

407. Borreria verticillata (L.) G. Mey.

408. Carapichea ipecacuanha (Brot.) L. Andersson.

409. Chomelia martiana Müll.Arg..

410. Chomelia obtusa Cham. \& Schltdl.

411. Coussarea capitata Benth. \& Hook. f.

412. Coussarea contracta (Walp.) Müll.Arg.

413. Coutarea hexandra (Jacq.) K. Schum.

414. Genipa americana L.

415. Hamelia patens.

416. Manettia cordifolia Mart.

417. Palicourea racemosa (Aubl.) Borhidi

418. Palicourea violacea (Aubl.) A.Rich.

419. Posoqueria latifolia Roem. \& Schult.

420. Posoqueria longiflora Aubl.

421. Psychotria bahiensis DC.

422. Psychotria bracteocardia (DC.) Müll. Arg.

423. Psychotria colorata (Willd. ex Roem. \& Schult.) Müll. Arg.

424. Randia armata (SW.) DC.

425. Sabicea grisea Cham.\& Schltdt.

426. Tocoyena bullata (Vell.) Mart.

427. Tocoyena formosa (Cham. \& Schltdl.) K. Schum.

\section{Rutaceae}

428. Ertela trifolia (L.) Kuntze.

429. Erythrochiton brasiliensis Nees \& Mart.

430. Esenbeckia pilocarpoides Kunth.

431. Metrodorea nigra A. St.-Hil.

432. Zanthoxylum monogynum A.St.-Hil.

433. Zanthoxylum rhoifolium Lam.

\section{Salicaceae}

434. Casearia zizyphoides Kunth

435. Xylosma ciliatifolia (Clos) Eichler

436. Banara brasiliensis (Schott) Benth.
$\mathrm{H}$

$\mathrm{H}$

$\mathrm{H}$

$\mathrm{H}$

V

$\mathrm{T}$

V

$\mathrm{V}$

$\mathrm{S} / \mathrm{T}$

$\mathrm{T}$

T

$\mathrm{T}$

SU

$\mathrm{SU}$

$\mathrm{S} / \mathrm{T}$

$\mathrm{S} / \mathrm{T}$

$\mathrm{S} / \mathrm{T}$

$\mathrm{S} / \mathrm{T}$

$\mathrm{S} / \mathrm{T}$

$\mathrm{S} / \mathrm{T}$

$\mathrm{S} / \mathrm{T}$

$\mathrm{V}$

$\mathrm{S} / \mathrm{T}$

$\mathrm{S}$

$\mathrm{S} / \mathrm{T}$

$\mathrm{S} / \mathrm{T}$

$\mathrm{S}$

$\mathrm{S}$

$\mathrm{S}$

$\mathrm{S} / \mathrm{T} / \mathrm{V}$

$\mathrm{V}$

$\mathrm{S}$

$\mathrm{SU} / \mathrm{S}$

$\mathrm{H} / \mathrm{SU}$

$\mathrm{S}$

$\mathrm{S} / \mathrm{T}$

$\mathrm{T}$

$\mathrm{T}$

$\mathrm{T}$

$\mathrm{T}$

$\mathrm{S} / \mathrm{T}$

$\mathrm{S} / \mathrm{T}$
K.M. Costa 9

Silva, TMC da; Almeida, K 21

Sobra-Leite M., Araújo J. F. 1402

Bento Pickel, 2155

Rita Lima, 671

Lima, R.; Barreto, R.; Barreto, R. CFPE-666

A.M. Miranda; et al. 519

Cassandra, L. 51375

Rodal, M.J.N., 1

V. C. Andrade 246

K. Almeida 114

A.M. Miranda; E.Araújo, S.I. Silva et

G.Gamarras 3605

Euripedes 18

Sobrinho, M.S. 549

Gomes, J. 6

Gomes, J.; Alves, M.; Melo, Y.; Araújo, A. \& Martins, S. 6

I. Pontual 1295

A.M. Miranda; E. Araújo, S.I. Silva et G. Gamarras 3598

Alves, J.L.H. de UFP5400

M.J.N. Rodal; T. M. C. Silva 772

Lopes, A.V. UFP21763

Pontual I. 1553

Cassandra L. s.n.

Maranhão E. 11

Sobrinho M. S. 39973

Primo L. M. 81499

Nadia T. L. 204

M.J.N. Rodal; A. G. Silva 800

K. Almeida; M.J.N. Rodal 72

Pinheiro K. 19

Sobrinho M. S. 528

Lopes A. V. 47335

Sobrinho M. 541

Sobrinho, M.S. 496

Siqueira-Filho, J.A.; Campelo, M.J.A. \& Lopes, A.V.F. 614

E. L. Sette Silva 36

Almeida, K. 48

Almeida, K. 58

Melo F. F. 238

K. Almeida, 90

F.A.A. Nepomuceno et al. 316

T.M.C. Silva; K. Almeida; M.F.L.R. Almeida;

M.V.P. Almeida; R. Almeida; R.F. Almeida \& S.S. Almeida 38

Sobrinho, M.S. 457 
438. Casearia sylvestris SW.

439. Prockia crucis P. Browne ex. L

\section{Sapindaceae}

440. Talisia cerasina (Benth.) Radlk.

441. Allophylus puberulus (Cambess.) Radlk.

442. Allophylus racemosus Sw.

443. Serjania hebecarpa Benth.

444. Allophylus edulis (A. St.-Hil., Cambess. \& A. Juss.)

445. Allophylus semidentatus (Miq.) Radlk.

446. Cupania racemosa (Vell.)

447. Cupania impressinervia Acev.-Rodr.

448. Paullinia pinnata L.

449. Paullinia trigonia Vell.

450. Serjania leptocarpa Radlk.

451. Serjania paucidentata DC.

452. Talisia retusa R.S. Cowan.

453. Talisia esculenta (A. St.-Hil.) Radlk..

\section{Sapotaceae}

454. Pouteria nordestinensis Alves-Araújo \& M. Alves

455. Chrysophyllum gonocarpum (Mart. \& Eichler ex Miq.) Engl.

456. Chrysophyllum rufum Mart..

457. Manilkara salzmannii (A. DC.) H. J. Lam.

458. Pouteria grandiflora (A. DC.) Baehni.

459. Pouteria ciliata A. Alves-Araújo

460. Pouteria glomerata (Miq.) Radlk.

461. Pouteria venosa (Mart.) Baehni.

462. Pradosia lactescens (Vell.) Radlk.

Smilacaceae

463. Smilax syphilitica Humb. \& Bonpl. ex. Willd.

\section{Solanaceae}

464. Solanum asperum Rich.

465. Brunfelsia uniflora (Pohl) D.Don.

466. Cestrum gardneri Sendtn.

467. Solanum americanum Mill.

468. Solanum paniculatum L.

469. Solanum rhytidoandrum Sendtn.

470. Solanum sycocarpum Mart. \& Sendtn.

Urticaceae

471. Cecropia concolor Willd.

472. Phenax sonneratii (Poir). Wedd.

\section{Verbenaceae}

473. Aloysia virgata (Ruiz \& Pav.) Juss.

474. Citharexylum myrianthum Cham.

475. Lantana canescens Kunth.

476. Lantana fucata Lindl.

477. Phyla betulifolia (Kunth.) Greene.

478. Stachytarpheta angustifolia (Mill.) Vahl.
$\mathrm{SU} / \mathrm{S} / \mathrm{T}$

$\mathrm{S} / \mathrm{T}$

$\mathrm{T}$

$\mathrm{S} / \mathrm{T}$

$\mathrm{S} / \mathrm{T}$

$\mathrm{S} / \mathrm{T}$

$\mathrm{T}$

$\mathrm{T}$

$\mathrm{T}$

V

$\mathrm{V}$

$\mathrm{V}$

$\mathrm{V}$

$\mathrm{T}$

$\mathrm{T}$

$\mathrm{S} / \mathrm{T}$

$\mathrm{T}$

$\mathrm{S}$

$\mathrm{T}$

$\mathrm{T}$

$\mathrm{T}$

$\mathrm{T}$

$\mathrm{S} / \mathrm{T}$

$\mathrm{T}$

V

$\mathrm{S} / \mathrm{T}$

$\mathrm{S}$

$\mathrm{S}$

$\mathrm{H}$

$\mathrm{S}$

$\mathrm{S}$

$\mathrm{S}$

$\mathrm{T}$

$\mathrm{SU} / \mathrm{S}$

$\mathrm{S} / \mathrm{T}$

$\mathrm{T}$

S

$\mathrm{S}$

$\mathrm{H}$

$\mathrm{S}$
Melo, Y.; Maciel, J.R. \& Amorim, D.A. 73

Sobrinho M.S. 493

E. L. Sette Silva, 31

M.S. sobrinho et al. 520

K. Almeida 128

Alunos de botânica sistemática (PEUFR).

Sobrinho, M.S. 520

Amorim, B.S.; Louzada, R. \& Costa-Lima, J.L. 1787

Almeida, K. 179

Cassandra, L. 51374

Santos E, G., Silva A. M. s.n.

Santos E, G., Silva A. M. s.n. Pickel B. 3114

Santos E. G., Silva A. M., s.n.

Amorim B. S. et al. 1790

Costa, K.C.; Silva, A.M. da; Silva, T.M.C. da;

Rodal, M.J.N.; Silva, A.M. da; Silva, T.M.C. da; Rodal, M.J.N. 212

K. Almeida, 13

Almeida, K.; Santos, E.; Santos, E. 13

I. Pontual 1392

Ameida Jr. E. B. 782

Chiappeta A. 33

T.M.C. da Silva; K. Almeida 67

T. M. C. da Silva; K. Almeida 45

Chiappeta A. 560

Miranda A. M. et al. 3618

Santos E. G., Silva A. M., s.n.

Sobral, M, 7291

Sobrinho, M.S. 460

Primo, L. s.n.

Maranhão E. 10

Pontual I. 1305

Sobrinho M. S. 313

Andrade V. C. 245

I. Pontual 1298

Lima, V. C., Andrade-Lima 07

Sobrinho, M.S. 456

Sobrinho, M.S. 535

Sobrinho M. 480

Sobrinho M. 300

Lima V. C. 46335

Sobral M. 7302

Violaceae 


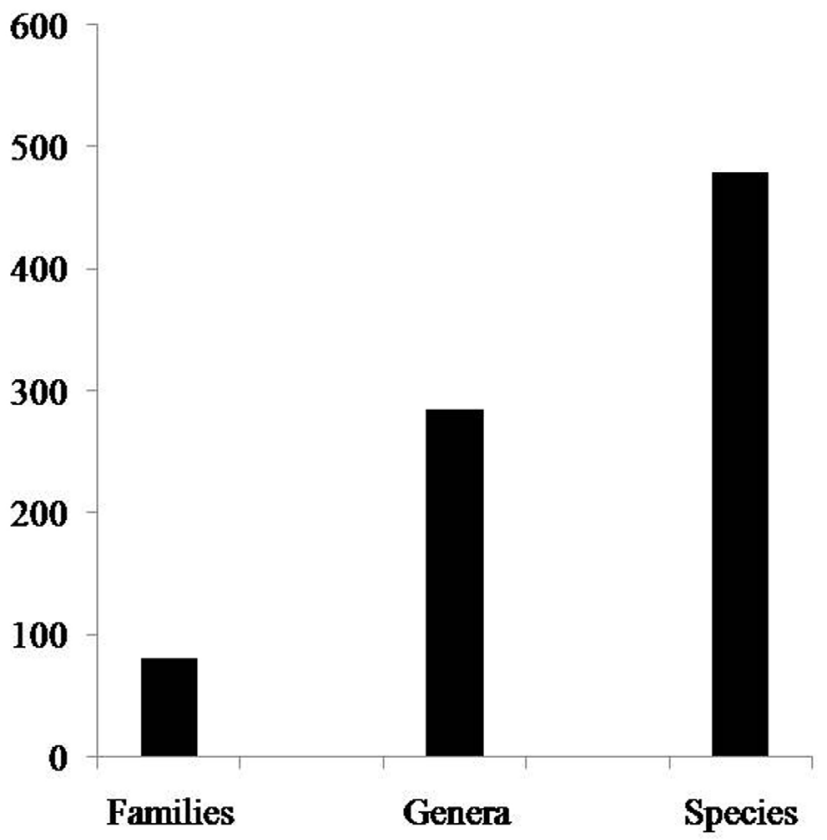

Figure 5. Numbers of families, genus and apecies from TES.

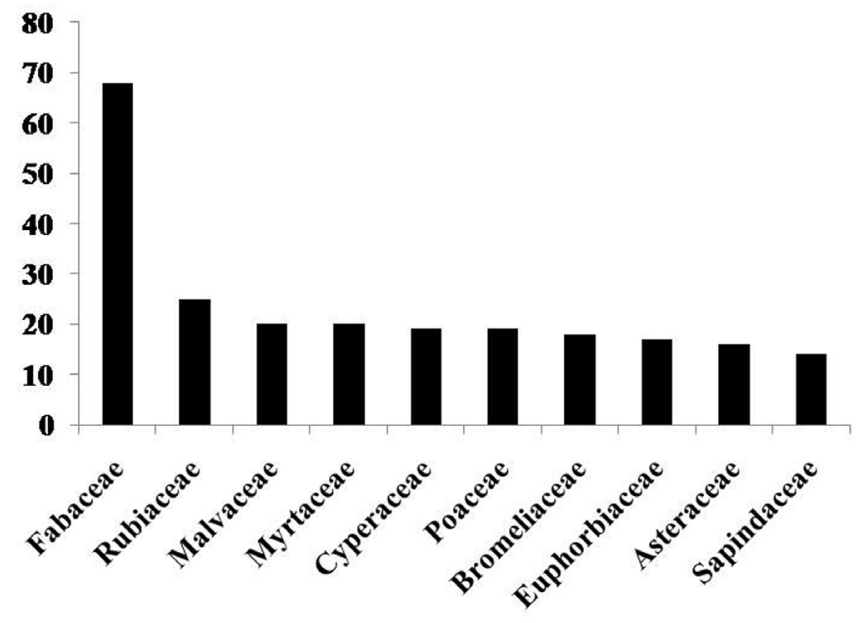

Figure 6. Ten most representative families in the TSE.

Among the genera listed for the TES, 21 (7.37\%) were best represented in terms of numbers of species: Cyperus L. (10 spp.), Eugenia L., Erythroxylum P. Browne, and Inga Mill. (7 spp. each), Aechmea Ruiz \& Pav. (6 spp.), Cordia L., Croton L., Miconia Ruiz. \& Pav., Myrcia DC., Paspalum L., Sida L., and Tillandsia L. (5 spp. each), followed by Adenocalymma Mart. ex Meisn. emend L.G. Lohmann, Aegiphila Jacq., Allophylus L., Coccoloba P. Browne, Desmodium Desv., Ruellia L., Rhynchospora Vahl, Pouteria Aubl., and Solanum L. (4 spp. each).

Herbaceous species were most commonly encountered (136 spp.; $28.39 \%$ of the total), followed by arboreal species ( 90 spp.; $18.79 \%$ ), lianas/climbers/vines (60 spp.; 12.53\%), shrubs 33 spp.; 6.89\%), and sub-shrubs (15 spp.; 3.13\%) (Figure 7). The families Cyperaceae (19 spp.), Poaceae (18), Bromeliaceae (18), Marantaceae (10), Orchidaceae (10), Asteraceae (8), and Commelinaceae (7) were the most species rich components of the herbaceous strata.

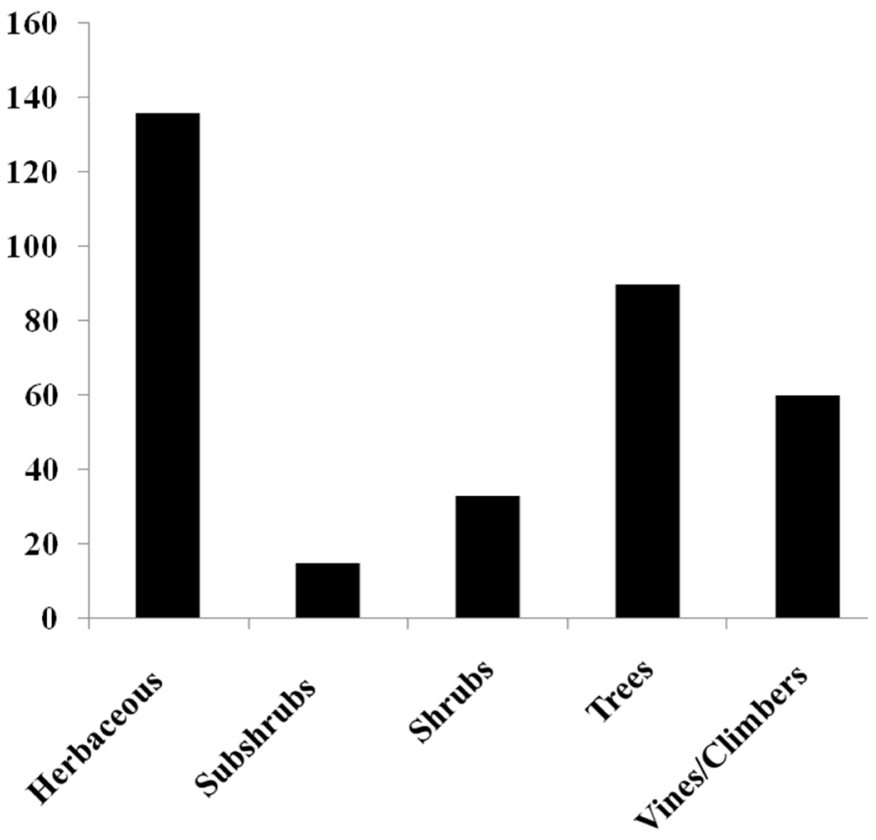

Figure 7. Numbers of species and their respective habits.

Among the most species rich arboreal families were: Fabaceae (25 spp.), Myrtaceae (13 spp.), Sapindaceae, and Sapotaceae (6 spp. each); among the richest species of shrub families were: Solanaceae and Rubiaceae (5 spp. each) and Melastomataceae (4 spp.); among the most species rich lianas/climbers/vines were: Fabaceae ( 9 spp.), Bignoniaceae (6 spp.), and Convolvulaceae and Sapindaceae (5 spp. each); and among the most species rich sub-shrubs were: Fabaceae and Acanthaceae (3 spp. each).

\section{Discussion}

The floristic profile of the main botanical families recorded in the present study, in terms of species richness, is similar to the data presented in studies undertaken in other regions of Lowland Rainforest and Dense Montana Rainforest in northeastern Brazil, reinforcing the importance of TES in Atlantic Forest conservation (Landim et al. 2015, Sobral-Leite 2011). The present work corroborates with the survey carried out by Landim et al. (2015) in the lowland Mata do Crasto forest in Sergipe State in listing Fabaceae (33 spp.), Rubiaceae (24), and Myrtaceae (23) as the most representative families; the species richness recorded for the Fabaceae family in TES, however, was more than double that (68 spp. vs 33) reported by Landim et al. (2015).

We also observed very similar results for angiosperms when comparing our results with those of Sobral-Leite (2011) from Mata do Estado, in the municipality of São Vicente Férrer, Pernambuco State. That area included vegetation of both the Caatinga (dryland) and Atlantic Forest phytogeographic domains, classified as Montane Rain Forest (sensu Veloso et al. 1991), and that author reported a predominance of the families Fabaceae (33 spp.), Myrtaceae (21) and Rubiaceae (20). Fabaceae is often the richest plant family in terms of species numbers even in the semiarid regions of Pernambuco with predominantly Caatinga vegetation and areas influenced by the Atlantic Forest and Cerrado (neotropical savanna), as was observed by Gomes et al. (2006) and Athiê-Souza et al. (2019). 
The Myrtaceae family in Lowland Rainforests in eastern Brazil, however, appears to be detached (Lima et al. 2012, Peixoto et al. 2008). Lima et al. (2012) carried out a floristic survey in the Carlos Botelho State Park in São Paulo, and reported that the richest families were Myrtaceae (85 spp.), Lauraceae (53), Fabaceae (37), and Rubiaceae (23). Peixoto et al. (2005) likewise cited Myrtaceae (185 spp.) as having the greatest species richness among the phanerogamic flora of the Linhares Reserve in Espírito Santo, followed by Fabaceae (109) and Rubiaceae (105).

In terms of plant genera, Cyperus L. (Cyperaceae), Eugenia L. (Myrtaceae), Erythroxylum P. Browne (Erythroxylaceae), and Inga Mill. (Fabaceae) stood out as having the largest numbers of species in our survey. Peixoto and Gentry (1990) noted that Myrtaceae species have significant ecological importance in forests along the Atlantic coast of Brazil, with great richness and abundance. Cordeiro and Loiola (2018), on the other hand, noted that Erythroxylaceae species frequently occur in seasonally dry climates, such as Savanna Steppe (Carrasco) and Deciduous Forests (dry forests).

Regarding the herbaceous strata, Poaceae has also been highlighted in terms of richness in lowland regions of the Atlantic Forest in the states of Sergipe and Rio Grande do Sul (Maraschin-Silva et al. 2009; Landim et al. 2015), as well as in areas of Semideciduous Seasonal Forest in the Atlantic Forest of Bahia State, and in Caatinga vegetation in Pernambuco (CostaJúnior et al. 2007; Alves et al. 2015; Athiê-Souza et al. 2019).

Among arboreal species, our results differ from Costa-Júnior et al. (2005), who studied the tree species in eight areas of Lowland Forest in Pernambuco State (Mata das Caldeiras, Mata do Zumbi, Mata de Tejipió, Mata do Resec Gurjaú, Mata dos Macacos, Mata do Curado, and Resec Dois Irmãos Forest) and highlighted the greatest similarity between Mata of Caldeiras and Mata of Curado; indicating Fabaceae, Moraceae, Anacardiaceae, and Annonaceae as the most representative families there.

In terms of shrub and sub-shrub species, those families having the highest numbers of species are generally cited as having the highest numbers of species in the shrub/sub-shrub components of the Atlantic Forest in northeastern Brazil (e.g., Amorim et al. 2005, Pereira \& Alves 2007, Alves et al. 2015) although occasionally in a different order. The family Fabaceae stands out in terms of species diversity in most of the surveys already mentioned, and also presents the most varied life forms - as seen in the present study area and confirmed by the observations of Gentry (1995) for dry neotropical forests. Queiroz (2009) noted that high plant habit diversity is related to the association of nitrogen fixing bacteria with root nodules, thus facilitating morphological adaptations and the occupation of a wide diversity of habits.

Considering the data available on the Flora do Brasil 2020 site and in floristic studies conducted in Pernambuco or northeastern Brazil (Sobral-Leite et al. 2010, Hatori 2009, Santos et al. 2013, Soares Neto 2014), we report here 44 new occurrences for Pernambuco State based on exsiccates deposited in herbaria. Most of the species reported here for the first time in Pernambuco were already known to occur in several states in northeastern Brazil, including: Abutilon ramiflorum A.St.-Hil. (BA); Bignonia binata Thunb. (CE); Combretum rotundifolium Rich. (CE); Mikania micrantha Kunth (CE); Nelsonia canescens (Lam.) Spreng. (BA, MA); Plathymenia reticulata Benth. (BA, CE, MA, PI); Pterocaulon alopecuroides (Lam.) DC. (BA, PB); Rivina humilis L. (BA, CE); Thunbergia fragrans Roxb. (BA); and Urena lobata L. (AL, BA, CE, MA, PB, SE).
Serjania leptocarpa Radlk (Sapindaceae), however, was the only species not mentioned for other states in northeastern Brazil (although identified in the states of Acre and Amazonas in the North, Rio de Janeiro in the Southeast, and Parana in the South) (Flora do Brasil 2020). S. leptocarpa was previously known only to Bolivia and Amazonas State in Brazil (Acevedo-Rodriguez 1993), in the Amazon rainforest, and in areas of the Atlantic Forest in northeastern and southern Brazil (Flora of Brazil 2020). Here, we recognize it as a new occurrence for Pernambuco State, which has been only poorly collected, but appears to prefer riverside habitats. According to Acevedo-Rodríguez (1990), the species of the genus Serjania that occur in humid areas generally have the widest distributions, which may be associated with the large number of rivers in the Amazon region that aid in propagule dispersal and help explain the adaptation of the genus to humid regimes. The same situation was observed for Serjania leptocarpa when herbarium label data were analyzed (Flora do Brasil 2020): that species is found in an Environmental Protection Area that surrounds the Negro River in the northern region of Brazil (considered the largest block of protected tropical forest in the world) (Ayres et al. 2005). There is also a wildlife corridor that crosses the Amazon-Solimões and Negro rivers (Ayres et al. 2005), contributing to gene flow through that region. S. leptocarpa occurs along the Turvo River in Paraná State, and it was recorded at the edges of the Tapacurá River in the TES in Pernambuco. According to the information available on GEOCAT, the species is classified as "of least concern" due to its extent of occurrence $\left(8.079,000 \mathrm{~km}^{2}\right)$ although, it can be classified as endangered due to its smaller area occupation of $120.000 \mathrm{~km}^{2}$.

Another new occurrence, Nelsonia canescens (Acanthaceae), also deserves mention here. The species has a curious distribution in Brazil, occurring from the northern part of the country in the states of Amazonas and Pará to the northeastern region with records for the states of Alagoas, Piauí, Rio Grande do Norte, and Bahia; it also occurs in the midwestern region of the country in Goiás State. Thus, Nelsonia canescens has a geographic distribution that includes areas of the Amazon and Atlantic forests, neotropical savanna, and semiarid forests - suggesting that the species has a disjunct distribution, as reported by Cavalcanti \& Tabarelli (2004) for some species with Amazonian-Northeastern occurrences.

The Tapacurá Ecological Station therefore holds a varied flora with high species richness and shares affinities with several other areas in northeastern Brazil. The rare species in this area have disjunct distributions between the Amazon rainforest and the Atlantic Forest. While our results have increased our knowledge of the flora of this region, additional efforts will still be needed for studies of other plant groups such as Bryophytes, Pteridophytes, and Gymnosperms that could reveal other new occurrences, and expand our knowledge of TES biodiversity.

\section{Acknowledgements}

We would like to thank the Estação Ecológica of Tapacurá for the support during the conducting of this work and Ednaldo José da Silva for helping in the fieldwork and images.

\section{Author Contributions}

Rafaela Alves Pereira-Silva: conceived the idea of the manuscript; structured, contributed to the writing of the text, and the interpretation of the results; conducted the fieldwork. 
Beatriz Rayrana de Araújo Gama: structured, contributed to the writing of the text, and the interpretation of the results; reviewed the species list, prepared the table; conducted the fieldwork.

Joésili Cristina Pereira de Oliveira: structured, contributed to the writing of the text, reviewed the species list.

Jone Clebson Ribeiro Mendes: structured, contributed to the writing of the text, and the interpretation of the results; conducted the fieldwork.

Jorge Irapuan de Souza Barbosa: reviewed the species list, confirmed the identification of species.

Sarah Maria Athiê-Souza: revised the manuscript.

Leidiana Lima dos Santos: structured, contributed to the writing of the text.

Margareth Ferreira de Sales: revised the manuscript.

Ana Luiza Du Bocage: also conceived the idea of the manuscript and revised the manuscript.

\section{Conflicts of Interest}

We, the authors, declare that we have no conflicts of interests related to the publication of this manuscript.

\section{References}

AYRES. J. M.; DA FONSECA, G. A. B. G.; RYLANDS, A. B.; H. L. QUEIROZ.; PINTO, L. P.; MASTERSON, D.; CAVALCANTI, R. B. 2005. Os Corredores Ecológicos das Florestas Tropicais do Brasil, 256, p.

ACEVEDO-RODRÍGUEZ, P. 1990. Distributional Patterns in Brazilian Serjania (Sapindaceae). Acta botânica brasílica v. 4, n.1:69-82.

ALMEIDA, A. V.; OLIVEIRA, M. A. B. 2009. A história da Estação Ecológica do Tapacurá (São Lourenço da Mata, PE) baseada no relatório de Vasconcelos Sobrinho de 1976. Recife.

ALVES, M.; OLIVEIRA, R. B.; TEIXEIRA, S. R.; GUEDES, M. L. S.; ROQUE, N. 2015. Levantamento florístico de um remanescente de Mata Atlântica no litoral norte do Estado da Bahia, Brasil. Hoehnea v. 42, n.3, p. 581-595.

AMORIM, A.M.; FIASCHI, P.; JARDIM, J.G.; THOMAS, W.W.; CLIFTON, B.C.; CARVALHO, A.M.V. 2005. The vascular plants of a forest fragment in southern Bahia, Brazil. Sida 21: 1726-1752.

ANDRADE, K. V. S. A., RODAL, M. J. N. 2004. Fisionomia e estrutura de um remanescente de floresta estacional semidecidual de terras baixas no Nordeste do Brasil. Rev. Brasil. Bot., v.27, n.3, p.463-474.

ATHIÊ-SOUZA, S. M., MELO, J. I. M., SILVA, L. P., SANTOS, L. L., SANTOS, J. S., OLIVEIRA, L. S. D., SALES, M. F. 2019. Phanerogamic flora of the Catimbau National Park, Pernambuco, Brazil. Biota Neotropica, n.19, v. 1, p. 1-27.

CAVAlCANTI, D.; TABARELli, M. 2004. Distribuição Das Plantas Amazônico-Nordestinas no Centro de Endemismo Pernambuco: Brejos De Altitude vs. Florestas De Terras Baixas. In: Brejos de Altitude Em Pernambuco e Paraíba História Natural, Ecologia E Conservação, 324 P.

CORDEIRO, L. S.; LOIOLA, M. I. B. 2018. Flora do Ceará, Brasil: Erythroxylaceae. Rodriguésia 69(2): 881-903.

COSTA JÚNIOR, ROBERTO F.; FERREIRA, RINALDO L. C.; RODAL, MARIA J. N.; FELICIANO, ANA L. P.; MARANGON, LUIZ C.; SILVA, WEGLIANE C. 2007. Florística arbórea de um fragmento de Floresta Atlântica em Catende, Pernambuco - Nordeste do Brasil. Revista Brasileira de Ciências Agrárias, v. 2, n. 4, p. 297-302.

CPRH - Agência Estadual de Meio Ambiente. 2017. Plano de Manejo do Refúgio de Vida Silvestre Mata de Tapacurá. Curitiba- PR, 216p.

DIAS, A. C. 2005. Composição florística, fitossociologia, diversidade de espécies arbóreas e comparação de métodos de amostragem na floresta ombrófila densa do Parque Estadual Carlos Botelho/SP-Brasil. Tese de doutorado. Doutorado em Recursos Florestais, USP. São Paulo.
FIDALGO, O.; BONONI, V. L. R. 1989. Técnicas de coleta, preservação e herborização de material botânico. São Paulo: Instituto de Botânica, 62p.

GENTRY, A.H. 1995. Diversity and floristic composition of neotropical dry forests. In Seasonally dry forests (S.H. Bullock, H.A. Mooney \& E. Medina, eds.). Cambridge University Press, Cambridge, p.146-194.

GOMES, A. P. S.; RODAL, M. J. N.; MELO, A. L. 2006 Florística da Vegetação arbustiva subcaducifólia de São José, Buíque, PE, Brasil. Acta Botânica Brasílica 20 (1):37-48.

HATTORI, E. K. O. 2009. Asteraceae da Estação Ecológica do Panga, Uberlândia, Minas Gerais. Tese de doutorado. Universidade Federal de Uberlândia. Programa de Pós-graduação em Ecologia e Conservação de Recursos Naturais. 169p.

IBGE. 2012. Manual técnico da vegetação brasileira ( $\left.2^{\mathrm{a}} \mathrm{ed}\right)$. Rio de Janeiro, 271p.

LANDIM, M.F., PROENC, A, C.E.B., SALES, A.B., MATOS, I.S. 2015. Floristic characterization of an Atlantic Rainforest remnant in Southern Sergipe: Crasto forest. Biota Neotropica, v. 15. n.1, 1-16.

LIMA, R. A. F.; SOUZA, V. C.; DITTRICH, V. O.; SALINO, A. 2012. Composição, diversidade e distribuição geográfica de plantas vasculares de uma Floresta Ombrófila Densa Atlântica do Sudeste do Brasil. Bitota Neotropica, v. 12, n 1 .

SOARES NETO. R. L. S.; CORDEIRO, L. S.; LOIOLA, M. I. B. 2014. Flora do Ceará, Brasil: Combretaceae Flora of Ceará, Brazil: Combretaceae. Rodriguésia v. 65, n. 3: 685-700.

MARASCHIN-SILVA, F., SCHERER, A., BAPTISTA, L. R. M. 2009. Diversidade e estrutura do componente herbáceo-subarbustivo em vegetação secundária de Floresta Atlântica no sul do Brasil. R. bras. Bioci., v. 7, n. 1, p. 53-65.

MELO, R. K. S. 2017. Estudo da Biodiversidade Como Ferramenta de Elaboração de um Plano de Manejo para Reserva Ecológica do Tapacurá, Pernambuco Brasil. Dissertação de Mestrado, mestrado em Gestão do Desenvolvimento Local Sustentável, UPE. Recife - Pernambuco.

MMM (2020), Ministério do Meio Ambiente. https://www.mma.gov.br/ (accessed in May 2020).

MOURA, G.J.B.; AZEVEDO-JÚNIOR, S.M.; EL-DEIR, A.C.A. 2012. A biodiversidade da Estação de Ecológica do Tapacurá: uma proposta de Manejo e conservação. Editora Nupeea.

OLIVEIRA, L. M. M. 2012. Estimativa da evapotranspiração real por sensoriamento remoto na bacia do rio Tapacurá - PE. Tese de doutorado, UFPE, Recife, Pernambuco.

PEREIRA, M. S.,ALVES, R. R. M. 2007. Composição Florística de um Remanescente de Mata Atlântica na Área de Proteção Ambiental Barra do Rio Mamanguape, Paraíba, Brasil. Revista de Biologia e Ciências da Terra, v.7, n.1.

PEIXOTO, A.L., GENTRY, A. 1990. Diversidade e composição florística da mata de tabuleiro na Reserva Florestal de Linhares (Espírito Santo, Brasil). Revista Brasileira de Botânica 13:19-25.

Peixoto, A. L.; Silva, I. M.; Pereira, O. J.; Simonelli, M.; Jesus, R. M.; Rolim, S. G. 2008. Tabuleiro Forests North of the Rio Doce: Their Representation in the Vale do Rio Doce Natural Reserve, Espírito Santo, Brazil. Memoirs of the New York Botanical Garden 100:319-350.

PENNINGTON, R. T., PRADO, D.E.; PENDRY, C.A. 2000. Neotropical seasonally dry forests and quaternary vegetation changes. Journal of Biogeography. Oxford, v. 27, p. 261-273.

QUEIROZ, L.P. 2009. Leguminosas da caatinga. Universidade Federal de Feira de Santana, Feira de Santana. 467 p.

RODAL, M. J. N.; LUCENA, M. F. A.; ANDRADE, K. V. S. A.; MELO, A. L. 2005. Mata do Toró: uma floresta estacional semidecidual de terras baixas no Nordeste do Brasil. Hoehnea v. 32. n.2: 283-294.

RODAL, M. J. N.; SALES, M. F.; SILVA, M. J.; SILVA, A. G. 2005. Flora de um Brejo de Altitude na escarpa oriental do planalto da Borborema, PE, Brasil. Acta bot. bras. 19(4): 843-858.

SANTOS, L. L.; SANTOS, L. L.; ALVES, A. S. A.; OLIVEIRA, L. S. D.; SALES, M. F. 2013. Bignoniaceae Juss. no Parque Nacional Vale do Catimbau, Pernambuco. Rodriguésia v. 64, n.3: 479-494. 
SOBRAL-LEITE, M.; CAMPELO, M. J. A.; SIQUEIRA-FILHO, J. A.; SILVA, SUZENE SILVA. 2010. Checklist das macrófitas vasculares de Pernambuco: riqueza de espécies, formas biológicas e considerações sobre distribuição. In: Biodiversidade, Potencial Econômico e Processos Ecofisiológicos em Ecossistemas Nordestinos, v. 2, 538 p.

SOBRAL-LEITE, M. 2011. Mata do Estado, São Vicente Férrer, Pernambuco, Brasil: Levantamento físicobiótico,Socioeconômico e Situação fundiária. In: Projeto, Apoio a Criação de Unidade de Conservação na Floresta Atlântica de Pernambuco, $46 \mathrm{p}$.

SOUZA, M. G.; WANDERLWY, M. G. L. 2000. Archmea Ruiz \& Pav. (Bromeliaceae) do estado de Pernambuco, Brasil. Acta Botânica Brasílica, v. 14, n. $1,77-97$.

THIERS, B. (2020) [continuously updated] Index Herbariorum: A global directory of public herbaria and associated staff. New York Botanical Garden's Virtual Herbarium. Available from: http://sweetgum.nybg.org/ science/ih/ (accessed January 2021).
VELOSO, H.P.; FILHO, L.C.O.; VAZ, A.M.S.F.; LIMA, M.P.M.; MARQUETE, R.; BRAZÃO, J.E.M.; FILHO, A.L.R.R.; DIAS, B.F.S.; PINTO, G.C.P.; MAGNAGO, H.; PEREIRA, J.B.S.; LIMA, J.C.A.; DAMBRÓS, L.A.; FURTADO, P.P.; KLEIN, R.M.; FILGUEIRAS, T.S.; BARROS, W.D. \& SILVA, Z.L. 1991. Manual Técnico da Vegetação Brasileira. Fundação Instituto Brasileiro de Geografia e Estatística - IBGE, Rio de Janeiro, Brasil.

Received: 06/04/2021

Accepted: 04/01/2022

Published online: 14/02/2022 